ñChemical equilibria studies using multivariate analysis methodsò Jaumot, J., Eritja, R., Gargallo, R. Anal. Bioanal. Chem., 399(6), 1983-1997 (2011). doi: 10.1007/s00216-010-4310-7

\title{
Chemical equilibria studies using multivariate analysis methods
}

\author{
Joaquim Jaumot, ${ }^{1, *}$ Ramon Eritja, ${ }^{2}$ Raimundo Gargallo ${ }^{1}$ \\ ${ }^{1}$ (UB-CSIC), Department of Analytical Chemistry, University of Barcelona, Diagonal 647, \\ 08028 Barcelona, Spain e-mail: joaquim@apolo.qui.ub.es \\ ${ }^{2}$ Institute for Research in Biomedicine, IQAC-CSIC, CIBER-BBN, Baldiri i Reixac 15, 08028 \\ Barcelona, Spain.
}

\begin{abstract}
Chemical multiequilibria systems can be monitored efficiently with the aid of spectroscopic techniques. Both hard- and soft-modeling are effective and powerful tools to extract chemical information from spectroscopic data. Recently, hybrid approaches that combine the flexibility of soft-modeling with the precise solutions provided by hard-modeling have been proposed. Here, we tested the performance of these three chemometric approaches for the analysis of several simulated data sets. In addition, experimental data recorded during the study of the acid-base equilibria of two DNA structures (G-quadruplex and $i$-motif) corresponding to two short sequences of the k-ras oncogene were studied. Finally, we also analyzed the interaction of the two DNA sequences with the model ligand TMPyP4. The results obtained from the analysis of these data sets may be useful to determine the most appropriate use of each approach. Whenever the presence of optically active interferences or unknown drifts can be neglected and a chemical model can easily be proposed and fitted, the hard-modeling method shows the best performance. If any of these conditions is not fulfilled, a hybrid modeling approach may be a better option because all the contributions (chemical and unknown) can be modeled and the ambiguities inherent to soft-modeling methods show minor effects.
\end{abstract}

Keywords. Multivariate data analysis. Chemical equilibria. Hard-modeling. Soft-modeling. Hybrid-modeling. k-ras

\section{Introduction}

Analytical instrumentation currently has the capacity to acquire large amounts of data rapidly. Traditionally, spectrophotometric monitoring of a chemical process has been carried out at just one or, exceptionally, at a few wavelengths. The combination of powerful detectors and the greater storage capacity of computers has, in many cases, allowed acquisition of the full spectrum of a sample, thus increasing the information registered [1]. Given this evolution, there is a demand for data analysis methods that can deal with these large data sets [2].

Univariate methods are used for this purpose whenever the signal is measured at only one wavelength: for example, the absorbance data recorded to characterize the progress of a 
reaction monitored at a single wavelength. In contrast, multivariate data are those recorded when the analytical signal is occuring simultaneously in multiple channels. In some cases, the analysis of univariate data can provide the desired chemical information. However, this may not be true for complex systems. In these cases, monitoring a process at one wavelength is not sufficient and ambiguities related to the proposed model, such as the determination of the number of species or their stoichiometry in the case of solution equilibria, are common. Multivariate resolution methods are powerful tools that can overcome these difficulties [1-3].

Multivariate resolution methods can be classified in two main groups. The first comprises those that fit the data to a previously proposed model and they are referred to as hardmodeling methods [1]. For example, for equilibrium studies, the model includes the stoichiometry of the species involved and the fulfillment of the Mass Action Law, together with an initial estimation of the equilibrium constants. An optimization process is then started whereby the proposed equilibrium constants are refined iteratively until a good fit is obtained between the calculated and the empirical data [4-6]. Moreover, the pure spectra for the species involved in the equilibria are determined. Hard-modeling methods may provide reliable solutions whenever a model can be easily proposed. However, these methods do not perform well when modeling interfering species or baseline drifts.

Some of these difficulties can be overcome using a second group of multivariate analysis techniques known as softmodeling methods, i.e., they do not use a chemical or physical model [7-9]. These methods seek to recover information without any assumption about the underlying model or law that governs the chemical reaction. In fact, soft-modeling approaches aim to propose a reliable model from the analysis of empirical data. However, in practice, this is difficult to achieve because of the presence of ambiguities (rotational or intensity), implying that many sets of equilibrium or kinetic constants and pure spectra can reproduce the initial data set with equal fit $[8,10]$. As a result of its capacity to apply constraints that contribute to providing chemical (physical, biological, $\cdots$ ) meaning to the pure mathematical solution, Multivariate Curve Resolution by Alternating Least Squares (MCR-ALS) is one of the most widely used soft-modeling methods [11-13]. In addition, the application of these constraints helps to minimize the effect of the rotational or intensity ambiguities [14-16].

Finally, in an attempt to combine the main advantages of hard- and soft-modeling approaches, recent years have witnessed the development of hybrid methods (or hard-/ softmodeling) [17-21]. In this mixed approach, the fulfillment of a hard-modeling constraint (kinetic model or Mass Action Law) is enforced during the optimization of a soft-modeling method (for instance, MCR-ALS). On the one hand, the hard-modeling constraint minimizes the ambiguity associated with the MCR-ALS solutions and provides values for the constants associated with the proposed model. On the other hand, the soft-modeling part of the 
optimization allows modeling contributions such as instrumental drifts or interfering species, which cannot be included in the model [17]. In this case, only the concentration profiles of those species involved in the process are forced to fulfill the proposed model, whereas the other components can be modeled using only the soft-modeling constraints. However, this approach also presents drawbacks, such as the selection of the model or the initial estimation for the values of the kinetic or equilibrium constants. This hybrid approach has been widely used to fit data to kinetic models but has been less used for equilibria studies.

Here, we compared the performance of these three approaches using simulated and experimental data corresponding to a range of solution equilibria. The simulated data sets were designed to check the weakness of each method by including optically inactive species or interfering species. The experimental data sets were obtained along the study of the solution equilibria of two DNA sequences (here named krasG1 and krasC1), which correspond to the guanine-rich and cytosine-rich strands of the nuclease-hypersensitive element respectively, located at the k-ras oncogene $[22,23]$. $k$-ras is one of the most frequently mutated oncogenes in human cancer. It encodes for a guanine nucleotide (GTP/GDP)-binding protein that acts as a signal transducer. This protein is anchored to the inner surface of the plasma membrane and serves as a molecular switch that transmits signals that influence cell growth and apoptosis to the nucleous. The $k$-ras gene may harbor a number of point mutations that yield a protein with an increased capacity to transmit signals for cell proliferation to the nucleus [23]. Several strategies have been proposed to address the expression of $k$-ras. Among these, studies have focused on the role of the nucleasehypersensitive site rich in guanines found in the regulatory regions of this gene [24, 25]. The krasC1 and krasG1sequences form structures that differ from the common Watson and Crick B-DNA. The former [25], which is rich in guanines, forms the known G-quadruplex structure stabilized by tetrads of four guanines (Scheme 1) [26, 27] while the latter may adopt the $i$-motif structure; a fourstranded structure that is stable in slightly acidic solutions because the protonation of several cytosine bases to form cytosine cytosine+ bonds is compulsory (Scheme 1) [28-30]. In this regard, the analytical study of the solution equilibria of krasG1 and krasC1 sequences may be useful to those researchers working on the control of $k$ ras transcription by means of DNA-ligand interactions.

Scheme 1 a Sequences studied in this study. Letters in bold indicate those that presumably participate in the tetrads which stabilize the G-quadruplex and i-motif structures, respectively. b Schematic structure of a Gquadruplex. c G-tetrad base pair. d Schematic structure of an i-motif. e Cytosine-cytosine + base pair. f Structural formula of TMPyP4. 
a)

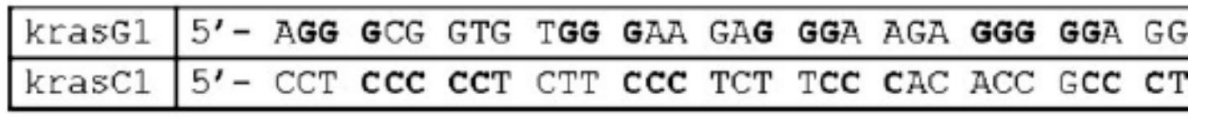

b)

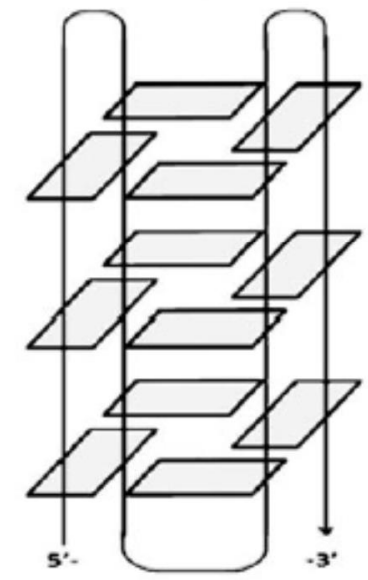

d)

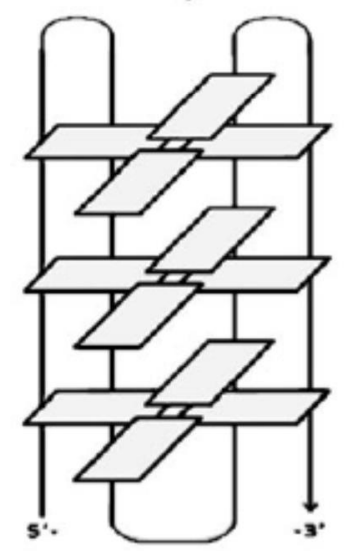

c)

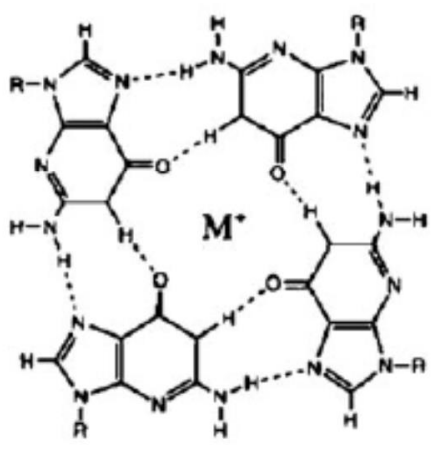

e)

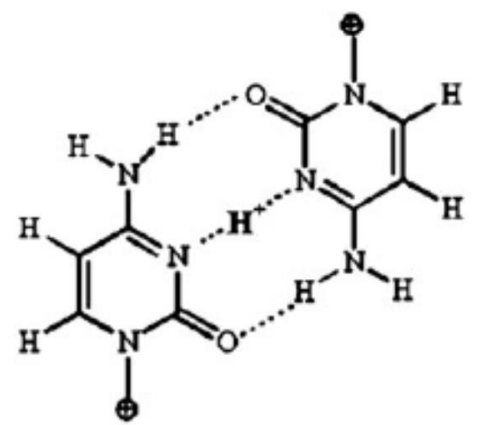

f)

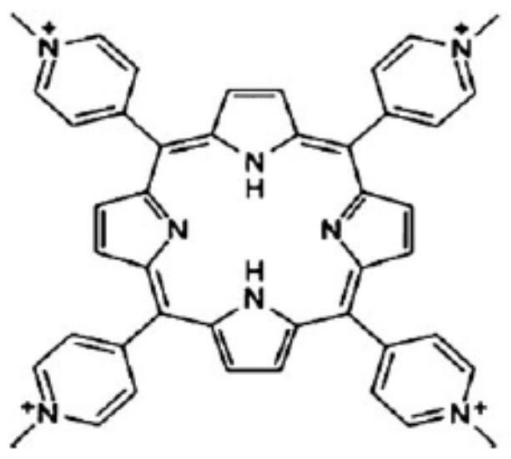

\section{Materials and methods}

Simulated data sets

Eight simulated data sets were generated to test the capacity of the resolution methods in diverse conditions. A summary of the data sets analyzed is shown in Table 1. Simulated data sets were built following Eq. 1: 
$\mathbf{D}_{\text {sim }}=\mathbf{C}_{\text {sim }} \mathbf{S}_{\text {sim }}^{\mathbf{T}}+\mathbf{N}$

where $\mathbf{D}_{\text {sim }}$ is the simulated data matrix, $\mathbf{C}_{\mathrm{sim}}$ is the matrix of simulated concentration profiles, $\mathbf{S}_{\text {sim }}^{\mathbf{T}}$ is the matrix of the pure simulated spectra of the different species and $\mathbf{N}$ is the matrix containing random noise added. The level of random noise added was $1 \%$ of the maximum intensity of the measured signal.

The concentration profiles and spectra used to build the acid-base simulated data sets are shown in Table 1 and Electronic Supplementary Material (Figure S1). In all cases, the analytical concentration was $2 \times 10 \overline{1} 6 \mathrm{M}$. Data set D1 simulates the acid-base titration of a H3A species in the $\mathrm{pH}$ range between 2.0 and 10.0, with the four chemical species optically active. Data set D2 is similar to D1 but, in this case, an interfering optically active species is present at $\mathrm{pH}$ values higher than 7. Data set D3 simulates the acid-base titration of H3A species, the species HAī being optically inactive. Finally, data set D4 is similar to D3 but contains an additional interfering optically active species at $\mathrm{pH}$ values higher than 7 . All these data sets have 81 rows (spectra at different $\mathrm{pH}$ values) and 481 columns (number of wavelengths).

The concentration profiles and spectra used to build the data sets simulating the DNAligand interaction are shown in Table 1 and Electronic Supplementary Material (Figure S2). Data set D7 simulates the titration of a species D (initial concentration $0.1 \mathrm{M}$ ) with a ligand (concentration ranging from 0.0 to $0.5 \mathrm{M}$ ). This set contains 51 spectra simulated at steps of ligand concentration of $0.01 \mathrm{M}$. The model is defined by a equilibrium constant that governs the formation of a 1:1 (D:L) species. Data set D8 simulates the formation of two interaction species DL and DL2. Data set D9 is similar to D8, the only difference being that species D is optically inactive. Finally, data set D10 is similar to D7 but contains an optically active interfering species at the end of the mole-ratio experiment.

Experimental data sets

Acid-base titrations were monitored by adjusting the $\mathrm{pH}$ of solutions containing the krasG1 or krasC1 sequences. This adjustment was performed by adding small volumes of $\mathrm{HCl}$ or $\mathrm{NaOH}$ stock solutions and spectra were recorded in a $\mathrm{pH}$ stepwise fashion. The titration of krasG1 (D5) was carried out over the $\mathrm{pH}$ range 2.13-8.52 at approximately $0.1 \mathrm{pH}$ intervals. At each pH, a full spectrum was registered from 220 to $360 \mathrm{~nm}$ (see Electronic Supplementary Material Figure S4a), thus generating a data matrix of 61 rows (values of $\mathrm{pH}$ ) and 141 columns (wavelengths). The titration of $\mathrm{krasC1}$ (D6) was done over the $\mathrm{pH}$ range 2.79-8.99 and, in this case, the matrix analyzed had 66 rows and 141 columns (see Electronic Supplementary Material Figure S4c). 
Table 1 Summary of the analyzed data sets

\begin{tabular}{|c|c|c|c|}
\hline $\begin{array}{l}\text { Data set } \\
\text { ID. }\end{array}$ & Type & Equilibria & Short description \\
\hline D1 & Simulated & Acid-Base & $\begin{array}{l}\text { Four chemical species optically } \\
\text { active without interfering } \\
\text { species } \\
\mathrm{p} K_{\mathrm{a} 1}=3, \mathrm{p} K_{\mathrm{a} 2}=5, \mathrm{p} K_{\mathrm{a} 3}=8\end{array}$ \\
\hline D2 & Simulated & Acid-Base & $\begin{array}{l}\text { Four chemical species optically } \\
\text { active with an interfering } \\
\text { species } \\
\mathrm{p} K_{21}=3, \mathrm{p} K_{\mathrm{a2} 2}=5, \mathrm{p} K_{\mathrm{a} 3}=8\end{array}$ \\
\hline D3 & Simulated & Acid-Base & $\begin{array}{l}\text { Four chemical species (three } \\
\text { optically active) without } \\
\text { interfering species } \\
\mathrm{p} K_{21}=3, \mathrm{p} K_{22}=5, \mathrm{p} K_{23}=8\end{array}$ \\
\hline D4 & Simulated & Acid-Base & $\begin{array}{l}\text { Four chemical species(three } \\
\text { optically active) with an } \\
\text { interfering species } \\
\mathrm{p} K_{\mathrm{a} 1}=3, \mathrm{p} K_{\mathrm{a} 2}=5, \mathrm{p} K_{\mathrm{2} 3}=8\end{array}$ \\
\hline D5 & Experimental & Acid-Base & krasGl titration \\
\hline D6 & Experimental & Acid-Base & krasCl titration \\
\hline D7 & Simulated & $\begin{array}{l}\text { Ligand-DNA } \\
\text { interaction }\end{array}$ & $\begin{array}{l}\text { Three chemical } \\
\text { species optically } \\
\text { active without } \\
\text { interfering species } \\
K_{1}=2,000 \mathrm{M}^{-1}\end{array}$ \\
\hline D8 & Simulated & $\begin{array}{c}\text { Ligand-DNA } \\
\text { interaction }\end{array}$ & $\begin{array}{l}\text { Four chemical species optically } \\
\text { active without interfering } \\
\text { species } \\
K_{1}=1,000 \mathrm{M}^{-1}, K_{2}=100 \mathrm{M}^{-1}\end{array}$ \\
\hline D9 & Simulated & $\begin{array}{l}\text { Ligand-DNA } \\
\text { interaction }\end{array}$ & $\begin{array}{l}\text { Four chemical species (three } \\
\text { optically active) without } \\
\text { interfering species } \\
K_{1}=1,000 \mathrm{M}^{-1}, K_{2}=100 \mathrm{M}^{-1}\end{array}$ \\
\hline D10 & Simulated & $\begin{array}{c}\text { Ligand-DNA } \\
\text { interaction }\end{array}$ & $\begin{array}{l}\text { Three chemical species } \\
\text { optically active with an } \\
\text { interfering species } \\
K_{1}=2,000 \mathrm{M}^{-1}\end{array}$ \\
\hline D11 & Experimental & $\begin{array}{l}\text { Ligand-DNA } \\
\text { interaction }\end{array}$ & TMPyP4:krasGl mole ratio \\
\hline D12 & Experimental & $\begin{array}{l}\text { Ligand-DNA } \\
\text { interaction }\end{array}$ & TMPyP4:krasCl mole ratio \\
\hline
\end{tabular}

Mole-ratio experiments to study the DNA-ligand interaction were performed by adding small volumes of oligonucleotide stock solution to an initial ligand solution. The spectrum was recorded $10 \mathrm{~min}$ after each addition of oligonucleotide. Experimental conditions were as follows: $25{ }^{\circ} \mathrm{C}, \mathrm{pH} 7.1$ for mole-ratio studies with krasG1 (data set D11) or $\mathrm{pH} 5.0$ with krasC1 (data set D12), and $150 \mathrm{mM}$ ionic strength (adjusted with $\mathrm{KCl}$ ). Experiments were carried out at CTMPyP4:CDNA ratios ranging from approximately 0 to 2. At each CTMPyP4:CDNA ratio a full spectrum was registered from 220 to $700 \mathrm{~nm}$. This procedure generated a data set of 25 
rows (molar ratios) and 481 columns (wavelengths) for D11 and 21 rows and 481 columns for D12 (see Electronic Supplementary Material Figure S5a and S5c).

Reagents

Thirty-two base-long oligonucleotides with sequences 5ôAGG GCG GTG TGG GAA GAG GGA AGA GGG GGA GG -3ô(krasG1) and 5ôCCT CCC CCT CTT CCC TCT TCC CAC ACC GCC CT-3ô(krasC1) were prepared on a 1 हmol scale using standard 2-cyanoethyl phosphoramidites (Cruachem Ltd.). Oligonucleotide synthesis was accomplished with an automatic DNA synthesizer (Applied Biosystems Mod. 392). Sequences were deprotected using standard protocols (concentrated ammonia, $55{ }^{\circ} \mathrm{C}$, and overnight). After deprotection, oligonucleotides were purified using purification cartridges in accordance with the manufacturer's instructions. Finally, purified oligonucleotides were desalted using Sephadex G25 columns to eliminate any residual salts. The length and homogeneity of these oligonucleotides was verified by $8 \mathrm{M}$ urea PAGE using Stain-All and reverse phase HPLC using X-Terra columns. DNA strand concentration was determined by absorbance measurements at $90{ }^{\circ} \mathrm{C}(260 \mathrm{~nm})$ using calculated extinction coefficients $(366,300$ and 241,200 for krasG1 and krasC1, respectively), and the nearest-neighbor method [31]. Before each experiment, the DNA solutions were previously heated to $90{ }^{\circ} \mathrm{C}$ for $10 \mathrm{~min}$ and allowed to reach room temperature. 5,10,15,20-Tetrakis-( $N$-methyl-4-pyridyl)- 21,23H-porphyrin tetratosylate (TMPyP4) was purchased from Porphyrin Systems Gbr (Lübeck, Germany) and used without further purification. Stock solution of porphyrin was prepared in water, stored at i $20{ }^{\circ} \mathrm{C}$ and diluted to working concentration immediately before use. An extinction coefficient value of $226,000(424 \mathrm{~nm})$ was used in the calculation of the concentration. $\mathrm{KCl}, \mathrm{KH}_{2} \mathrm{PO}_{4}, \mathrm{~K}_{2} \mathrm{HPO}_{4}$, $\mathrm{HAcO}, \mathrm{HCl}$, and $\mathrm{NaOH}$ (a.r.) were purchased from Panreac (Spain). MilliQ® water was used in all experiments.

Apparatus

Absorbance spectra were recorded on an Agilent HP8453 diode array spectrophotometer. The temperature was controlled via an 89090A Agilent Peltier device. A Hellma quartz cell (1.0 path length and 3,000 عl volume) was used. $\mathrm{pH}$ values were measured with an Orion SA $720 \mathrm{pH} / \mathrm{ISE}$ meter and micro-combination $\mathrm{pH}$ electrode (Thermo). All experiments were carried out without removing the measurement cell from the cell holder, thereby taking advantage of the stirrer incorporated to the Peltier device and minimizing the experimental error.

Data analysis 
Data obtained from the spectroscopic monitoring of a process are grouped in a data matrix $\mathbf{D}$ whose $m$ rows are the number of spectra recorded during the process and whose $n$ columns are the total number of wavelengths measured [2]. In general, the goal of all the resolution methods is the decomposition of the data matrix $\mathbf{D}$ following Eq. 2:

$\mathbf{D}=\mathbf{C} \mathbf{S}^{\mathbf{T}}+\mathbf{E}$

where $\mathbf{C}$ is a $m \times k$ matrix, $\mathbf{S}^{\mathbf{T}}$ is a $k \times n$ matrix, and $\mathbf{E}$ is a $m \times n$ matrix. $k$ is the number of factors directly related to the chemical species present during the process, i.e., $k$ corresponds to the optically active species. Therefore, columns of $\mathbf{C}$ describe the concentration profiles for the $k$ different species during the process. Rows of $\mathbf{S}^{\mathbf{T}}$ matrix describe the pure spectra for each of these $k$ components. Finally, $\mathbf{E}$ is the matrix of residuals not explained by the multiplication of $\mathbf{C}$ and $\mathbf{S}^{\mathbf{T}}$. However, the solution of Eq. 2 for $\mathbf{C}$ and $\mathbf{S}^{\mathbf{T}}$ is ambiguous if no additional information is available. In other words, there is a rotational and/or scale (intensity) freedom in the solutions of Eq. 2. Therefore, additional information is required to obtain a reliable solution. The mathematical procedures to decompose $\mathbf{D}$ can be split into two groups, namely hard- and soft modeling methods. The first involves those approaches that fit the data to a previously proposed model while the second group involves those methods that do not apply these models along the calculation.

Hard-modeling methods

In the case of hard-modeling methods, a model is applied to guide the decomposition of matrix D following Eq. 2. As an example, in the case of acid-base equilibria, the model includes the fulfillment of the Mass Action Law and the stoichiometry of the species formed, as well as initial trial values for the equilibrium constants [5, 17, 32]. These values are used to build an initial estimation of the concentration profiles included in $\mathbf{C}$. Once this estimation is obtained, a least squares fit is performed to obtain the pure spectra in $\mathbf{S}^{\mathbf{T}}$ that best match the concentration profiles in $\mathbf{C}$ and the data in $\mathbf{D}$. A residual matrix $\mathbf{E}$ is then calculated from the difference between the data in $\mathbf{D}$ and the reproduced data matrix. The optimization procedure continues by tuning the stoichiometry of the proposed species until a desired level of residuals $\mathbf{E}$ is reached. A more detailed explanation of the mathematical basis of the algorithms used in these calculations can be found in Maeder and Zuberbühler [4].

Soft-modeling methods 
Soft-modeling methods do not use a model in the calculation [8]. Several methods solve Eq. 2 iteratively by a least squares algorithm that calculates concentration $\mathbf{C}$ and pure spectra $\mathbf{S}^{\mathbf{T}}$ matrices, optimally fitting the experimental data matrix $\mathbf{D}$ using the proposed number $k$ of species. MCR-ALS is one of these soft-modeling methods and has been extensively applied to the analysis of different types of data. A detailed description of this approach can be found therein [11, 13, 33]. The iterative optimization requires initial estimations of either $\mathbf{C}$ or $\mathbf{S}^{\mathbf{T}}$, which can be obtained from pure variable detection or local rank analysis methods. In this study, we used an initial estimation of the spectra profiles by applying a pure variable approach based on the SIMPLISMA method [34]. When an initial estimation of ST is considered, the next step is the estimation of the $\mathbf{C}$ matrix. During this calculation, some constraints can be applied to give a meaning (physical, chemical, biological $\cdots$ ) to the pure spectra and/or concentration profiles mathematically calculated [12]. Examples of these constraints are the non-negativity, unimodality of the profiles, and concentration closure (for closed systems). Finally, residuals (E) are calculated from the $\mathbf{C}$ and $\mathbf{S}^{\mathbf{T}}$ matrices obtained and the process is repeated until a convergence criterion is fulfilled. As shown, the resolution process in MCR does not use a model, and only a few constraints are applied along the alternating least squares optimization to give a meaning to the final solutions. However, there is still not full confidence in the reliability of the results, especially for systems showing a high degree of spectral and/or concentration overlap, which causes the ambiguities discussed above to strongly influence the results obtained.

A number of different strategies can be used to minimize the effect of the ambiguities, such as the application of constraints (especially selectivity constraints) or the simultaneous analysis of several experiments.

Hybrid-modeling methods

In order to overcome the ambiguities inherent to soft-modeling methods and the rigidity characteristic of hard-modeling ones, intermediate or hybrid approaches attempt to combine the best of each approach. One of these hybrid methods is based on the previously developed softmodeling MCR-ALS approach. Now, a hard-modeling related constraint (for instance, an equilibrium [18] or kinetic model [17]) must be fulfilled along the ALS optimization. This constraint is applied by selecting the appropriate concentration profiles in C, i.e., those involved in the chemical process, as an input for a non-linear hard-modeling fitting. This procedure allows the minimization of the ambiguities of the related concentration and spectra profiles and, consequently, an improvement in the quality of the results obtained together with the recovery of the model parameters, such as equilibrium or rate constants. In addition, the soft-modeling part of the algorithm allows the modeling of any interfering optically species or component, 
such as baseline drift. A detailed description of the implementation of the constraint and the non-linear fitting algorithms that can be used during the hard-modeling optimization can be found in the literature $[9,17,18,35]$. This hybrid method also requires an initial estimation of either $\mathbf{C}$ or $\mathbf{S}^{\mathbf{T}}$ profiles and, in all cases, the same initial estimations as in the pure soft-modeling resolution are used.

The quality of the results obtained with the three different methods was quantified from the concentration and spectral profiles resolved. Hence, we calculated a dissimilarity parameter from the comparison of the simulated and calculated profiles [36]. This parameter corresponds to the sine of the angle between the two compared profiles, ranging its magnitude from 0 (when the profiles compared are equal) to 1 (when the profiles compared differ completely).

In all cases, Matlab® R2008a [37] was used in the calculations. Soft- and hybridmodeling calculations were performed using the MCR-ALS programs [13, 14, 18] while those for hard-modeling were done using the Equispec program [32].

Results and discussion

The results obtained with the methods are separated on the basis of the chemical equilibria studied.

Simulated data sets corresponding to acid-base equilibria

The concentration profiles and spectra resolved for the four simulated data sets are presented in Fig. 1 and the values obtained for the equilibrium constants are shown in Table 2. On the basis of these results, the behavior of the three resolution approaches can be compared.

First, we examined the simplest case, in which all species were optically active and there were no interfering species (D1). Analysis with a hard-modeling method proposed a model with four species, and pKa values of 2.5, 6.0, and 9.0. The soft-modeling resolution was done using non-negativity constraints for concentration and spectra profiles and a closure constraint (the sum of the concentrations at each point must be lower or equal to $2 \times 10^{\overline{1} 6} \mathrm{M}$ ). Finally, analysis with the hybrid method using the non-negativity constraints and the hardmodeling constraint defined by a model with a total concentration of $2 \times 10^{i} 6 \mathrm{M}$, four species and $\mathrm{pK}_{\mathrm{a}}$ values of 2.5, 6.0, and 9.0.

The concentration profiles and spectra calculated with the hard-modeling method and with the hybrid method were almost identical to the spectra used in the simulation of the data (Fig. 1a and b, and Tables 2 and Electronic Supplementary Material Table S1). The dissimilarity values for this data set using the hard or hybrid-modeling methods were near 0 , thereby indicating a good correlation with the simulated profiles. The solutions obtained by the pure soft-modeling method showed a slightly different behavior. In this case, more substantial 
differences between simulated and calculated profiles were observed. In addition, we studied the impact of the rotational ambiguities in this simplest case. As a result of the simplicity of the data (without interferences and with good resolution conditions), the effects of the ambiguities can be considered negligible except for the more basic species (see Electronic Supplementary Material Figure S3).

Fig. 1 Results from the analysis of the acid-base simulated data sets. a Resolved concentration and b spectra profiles for data set D1. c Resolved concentration and d spectra profiles for data set D2. e Resolved concentration and f spectra profiles for data set D3. g Resolved concentration and h spectra profiles for data set D4. Solid lines simulated profiles of spectroscopically active species, circle lines simulated profiles of spectroscopically inactive species, dashed lines hard modeling profiles, dotted lines soft-modeling profiles, dash-dot lines hybrid-modeling profiles.
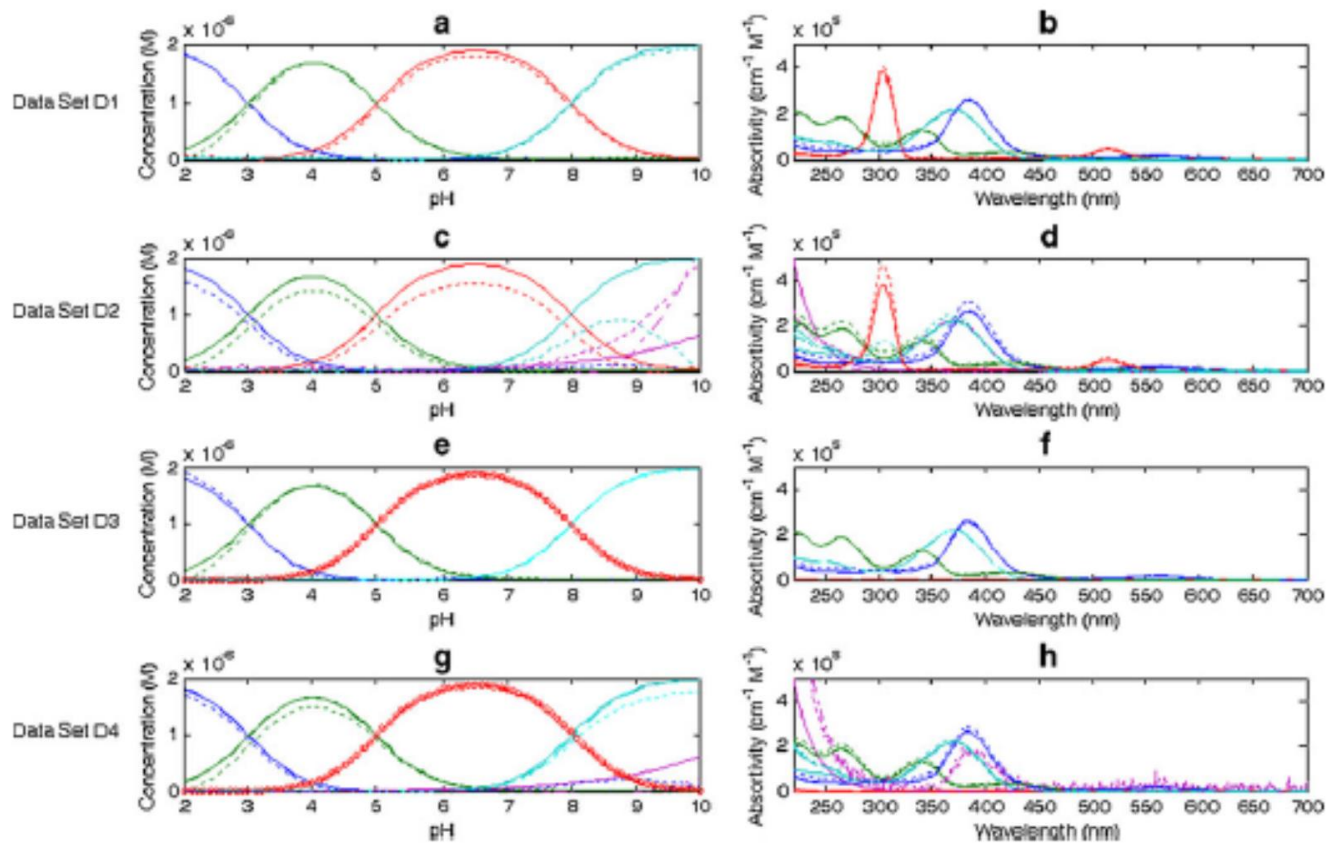

Table 2 Equilibrium (grotonstion) wnstants for the data sets simulating acid- base trations as calculated with the three methods tedal. Standard deviabion valuas ane in brackets

\begin{tabular}{|c|c|c|c|c|}
\hline & & Hard-modeling & Sofl-modeling & Hylrid-modeling \\
\hline \multirow[t]{3}{*}{ D1 } & $\mathrm{p} K_{\mathrm{a} 1}$ & $2998(0.002)$ & 3.034 & $2.9937(0.0008)$ \\
\hline & $\mathrm{p} K_{\mathrm{a}}$ & $5.002(0.001)$ & 5.040 & $5.0020(0.0008)$ \\
\hline & $\mathrm{p} K_{\mathrm{m}}$ & $7.9988(0.0008)$ & 7.992 & $8.0029(0.0008)$ \\
\hline \multirow[t]{3}{*}{ D2 } & $\mathrm{p} K_{\mathrm{a} 1}$ & $3.00(0.01)$ & 3.034 & $2.9924(0.0009)$ \\
\hline & $\mathrm{p} K_{\mathrm{a} 2}$ & $4.996(0.008)$ & 5.030 & $5.0007(0.0009)$ \\
\hline & $\mathrm{p} K_{\mathrm{ma}}$ & $8.031(0.006)$ & - & $8.0012(0.0009)$ \\
\hline \multirow[t]{3}{*}{ D3 } & $\mathrm{p} K_{\mathrm{a} 1}$ & $2999(0.001)$ & 3.043 & $2.990(0.002)$ \\
\hline & $\mathrm{p} K_{\mathrm{a} 2}$ & $4.995(0.001)$ & $-{ }^{*}$ & $5.022(0.003)$ \\
\hline & $\mathrm{p} K_{\mathrm{as}}$ & $8.0005(0.0007)$ & $-{ }^{*}$ & $8.017(0.003)$ \\
\hline \multirow[t]{3}{*}{ D4 } & $\mathrm{p} K_{\mathrm{a} 1}$ & $3.01(0.02)$ & 3.045 & $2.994(0.003)$ \\
\hline & $\mathrm{p} K_{\mathrm{m} 2}$ & $4.99(0.01)$ & 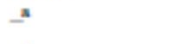 & $5.028(0.004)$ \\
\hline & $\mathrm{p} K_{\mathrm{al}}$ & $8.06(0.01)$ & $=$ & $8.029(0.004)$ \\
\hline
\end{tabular}

${ }^{\mathrm{p}} K_{\mathrm{a}}$ values obtained from soft-modeling method (MCR-ALS) are

detarmined from the crossing points of the concentration profiles 
The results from the analysis of D2 are shown in Fig. 1c and d. In this case, hard-modeling was carried out as in the analysis of D1 because the interfering species cannot be modeled. In contrast, soft-modeling was carried out for five species (detected by singular value decomposition, SVD [38]) and applying the non-negativity and closure (only for the four species involved in the equilibria) constraints. Finally, the hybrid analysis was performed for the five species in which only four participated in the model. The soft-modeling approach did not fully resolve the most basic and the interfering species, a finding that was also denoted in dissimilarity values (Electronic Supplementary Material Table S1). The results from the analysis of D2 differed depending on the analytical method (hard or hybrid) applied. Hardmodeling provided a solution (with correct $\mathrm{pKa}$ values) without taking into account the presence of the interference. Consequently, the resolved spectra differed from the simulated ones, in spite of the observation that the concentration profiles calculated were acceptable. Regarding hybridmodeling, although the profiles of the most basic and the interfering species were both fully resolved, the possibility of modeling the interfering species allowed a better calculation of the concentration and spectra profiles for the four species and for the interference.

Data set D3 was similar to D1 but with the property that the third species was not optically active. Therefore, the hard model was modified to take into account that this species did not absorb while the rest of the conditions remained unaltered. In the case of the softmodeling approach, SVD detected only three species and therefore the resolution procedure was done for this number of species. Finally, for the hybrid approach, the resolution was performed for the three optically active species. However, the postulation of the model with the four species (only three will show a spectrum) also allowed the modeling of the optically non-active species and recovery of the $\mathrm{pK}_{\mathrm{a}}$ values. The results obtained showed a great concordance between the three approaches used (Fig. 1e and f). As in the previously described data sets, the results from the soft-modeling approach differed slightly from those obtained with the other two methods. Hence, the second and third pKa values were not accurately determined because the concentration profile of the third species (non-optically active) was not correctly calculated. In the case of hard-modeling and hybrid-modeling, the concentration and spectra profiles calculated were practically identical to the simulated ones, despite the fact that the dissimilarity values were slightly worse in the case of the latter approach.

Finally, data set D4 included the three optically active species (as in D3) and one interfering optically active species (as in D2). Consequently, the hard-modeling approach used a similar model to that described for the analysis of D3 but did not consider the interfering species. SVD detected four species corresponding to the three optically active chemical species and the interfering species. Therefore, the soft-modeling resolution was carried out using four species and applying a closure constraint (equal to $2 \times 10^{i}{ }^{6} \mathrm{M}$ ) only for three of the four species detected. Finally, the hybrid approach was performed also for four species, of which only three 
were included in the hard model. The results obtained are shown in Fig. 1g and h. Hardmodeling showed a good recovery of the concentration profiles with correct $\mathrm{pKa}$ values. However, the spectral profiles were poorly calculated, especially that corresponding to the most basic species because it was clearly modified by the spectral profile of the interfering species. The concentration profiles calculated by the soft-modeling approach showed good correlation with the simulated ones, despite the fact that the interfering species was not correctly modeled. This observation is also reflected in the pure spectra calculated in which this interfering species showed an absorption band at approximately $400 \mathrm{~nm}$. This band was assumed to be an artifact of the optimization procedure. The resolved spectra of this interfering species showed a high amount of noise, a finding that indicates uncertainty in the resolved profile. Again, this resolution allowed a reliable calculation only of $\mathrm{pK}_{\mathrm{a}} 1$ because it was not possible to correctly calculate the concentration profile of the optically inactive species. Finally, the hybrid approach provided a combination of the results obtained by the other two methods. Hybrid-modeling allowed the accurate calculation of the $\mathrm{pK}_{\mathrm{a}}$ values because the concentration profile of an optically inactive species in the hard model was recovered. Furthermore, the soft-modeling part of the algorithm allowed the modeling of the behavior of the interfering species, thereby notably improving the resolution of the other species. In addition, the application of the hard model also improved the resolution of the interference because its spectrum was better resolved without the band at $400 \mathrm{~nm}$ and there was a non-appreciable amount of noise (Fig. 1h).

The results from the analysis of these data sets are useful to determine the circumstances in which each approach should be applied. In the case of simple systems (without baseline drifts or interfering species), the best results are obtained by hard-modeling. However, when some of these conditions are not fulfilled, the results from the hybrid-modeling method are the most reliable.

Data sets recorded along the experimental acid-base study of krasG1 and krasC1

The first experimental example corresponds to the $\mathrm{pH}$ titration of the krasG1 sequence. This sequence is composed by the nucleosides guanosine ( $\mathrm{pK}_{\mathrm{a}}$ values equal to 1.6 and 9.2), thymidine $\left(\mathrm{pK}_{\mathrm{a}}\right.$ value around 9.7), adenosine $\left(\mathrm{pK}_{\mathrm{a}}\right.$ value around 3.5), and cytidine $\left(\mathrm{pK}_{\mathrm{a}}\right.$ value around 4.5) [39]. On the basis of the $\mathrm{pK}_{\mathrm{a}}$ values of guanosines, dramatic modifications in the Gquadruplex structure of the oligonucleotide over the $\mathrm{pH}$ range studied were not expected because of protonation or deprotonation of guanine bases in the tetrad (Scheme 1) [40]. However, the presence of adenosines and cytidines (which are not involved in the stability of tetrads because they are present at the loops) may produce acid-base equilibria without disrupting the G-quadruplex structure. 
The results (concentration and spectra profiles) for the three resolution approaches are shown in Fig. 2. First, data set D5 was analyzed by means of the MCR-ALS soft-modeling method. This approach was chosen because does not require the proposal of a model and the resolution could provide knowledge useful to define the model needed in the hard- or hybridmodeling methods. Analysis with SVD indicated the presence of three main optically active species. The concentration profiles calculated by MCR-ALS showed a major species at $\mathrm{pH} 7$, which was assigned to the G-quadruplex structure in which all the bases were in their neutral form. At $\mathrm{pH}$ values lower than 7, a new species appeared, the formation of which peaked at around $\mathrm{pH}$ 4.5. According to the $\mathrm{pH}$-transition midpoint, this species was related to the protonation of cytidine. Finally, at strongly acidic conditions, a third species appeared. This species was related to the protonation of the adenosine of the sequence (the pH-transition midpoint was around 3.5).

Fig. 2 Results from the analysis of the acid-base experimental data sets. a Resolved concentration and b spectra profiles for the krasG1 data set (D5). c Resolved concentration and d spectra profiles for the krasC1 data set (D6). dashed lines hard-modeling profiles, dotted lines soft-modeling profiles, dash-dot lines hybrid-modeling profiles.
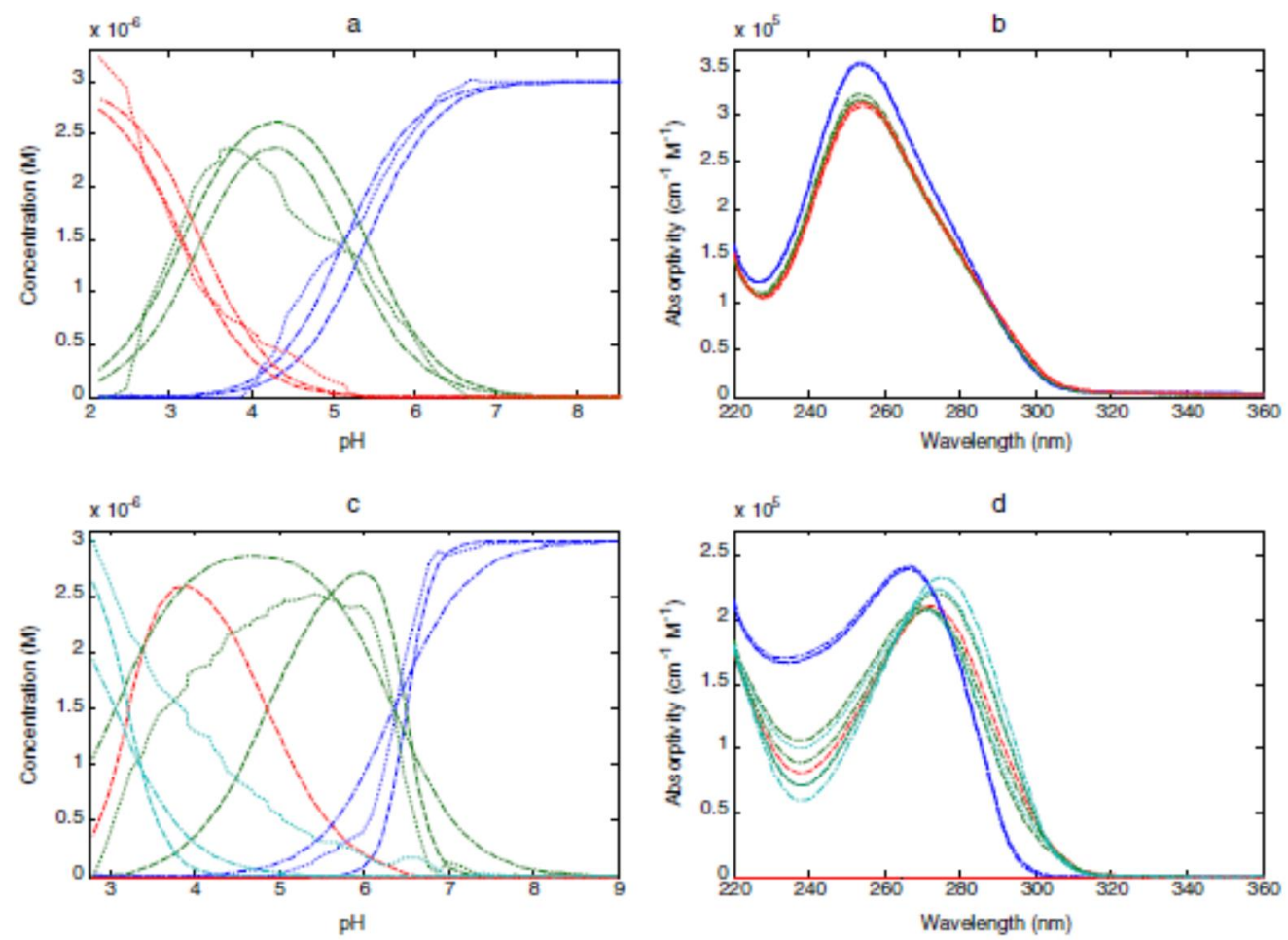

Using the information obtained with the soft-modeling analysis, we performed the resolution using the pure hardmodeling method. Thus, a model with three species was proposed that correctly fitted the data. We also tested other models with more and fewer species and/or distinct stoichiometries but these did not significantly improve the fit. Finally, we analyzed the data using the hybrid-modeling approach and the results were consistent with those obtained using a model with three species. This similarity can be seen in the resolved profiles of Fig. 2 
and in the recovered $\mathrm{pK}_{\mathrm{a}}$ values shown in Table 3 . The results obtained by the hard- and hybridmodeling methods were similar. In contrast, those results obtained by the soft-modeling method shower greater differences especially regarding the resolved concentration profiles (Fig. 2a). However, in all cases, our results were qualitatively consistent with those obtained previously for one of the two G-quadruplex structures present in the c-kit oncogene [41].

The second system studied here corresponded to the $\mathrm{pH}$ titration of $\mathrm{krasC} 1$, a sequence comprising mostly cytidine and thymidine. In accordance with the prior knowledge of similar sequences [42-46], the variation of $\mathrm{pH}$ was expected to be relevant because the DNA structure forms as a result of the half-protonation of cytidine (Scheme 1).

Analysis with SVD indicated the presence of three main optically active species. The distribution diagram calculated with MCR-ALS (Fig. 2c) showed two pH-midpoint transitions located around 3.8 and 6.4. A steep slope was observed for the concentration profiles around the transition at $\mathrm{pH}$ 6.4. This slope can be associated with a cooperative effect, in which the protonation of a cytosine base to yield the cytosine cytosine $^{+}$base pair strongly promotes the protonation of neighboring bases [47, 48].

Table 3 Equilibrium (protonation) constants calculated for the experimental data corresponding to the acid-base equilibria of lasal (data set D5) and krasc1 (data set D6) sequences as calculated with the three methods lested

\begin{tabular}{|c|c|c|c|c|c|c|}
\hline & \multicolumn{3}{|l|}{ Krasi1 } & \multicolumn{3}{|l|}{ KrasC1 } \\
\hline & Hard-modeling & Sott-modeling ${ }^{\mathrm{a}}$ & Hybrid-modeling & Hard-modeling & Sof-modeling & Hybrid-modeling \\
\hline $\mathrm{p} K_{\mathrm{a} 1}$ & $3.2(0.1)$ & 3.094 & $3.4(0.2)$ & $2.7(0.1)$ & 3.76 & $3.0(0.1)$ \\
\hline $\mathrm{p} K_{\mathrm{m}}$ & $5.42(0.08)$ & 4.930 & $5.2(0.2)$ & $4.64(0.07)$ & 6.41 & $6.4(0.1)$ \\
\hline $\mathrm{p} K_{\mathrm{a}}$ & & & & $6.46(0.03)$ & & \\
\hline
\end{tabular}

Standard deviation values are in brackets

${ }^{\mathrm{p}} K_{\mathrm{a}}$ values obtaind from sof modeling method (MCRALS) are deterninal from the crossing points of the oncentration profiles

The resolution using the hard-modeling method differed slightly from that described for krasG1. In this case, the models applied for the analysis of acid-base data corresponding to monomers (those which consider the uptake of one proton in each acid-base equilibrium) failed. The model that best fitted the experimental data was that which considered the presence of up to four species (Table 3, Electronic Supplementary Material Figure S4). The major acid-base species in neutral solution was explained as the neutral krasC1 sequence, where all bases were deprotonated. At $\mathrm{pH}$ below 7, a new species appeared. We attributed this species to an $i$-motif structure. The sharp absorbance change over the $\mathrm{pH}$ range 6 to 7 was successfully modeled when the uptake of protons was set to 3 . This value differed considerably from that observed for monomers and was clearly related to a cooperative transition [47-49]. A gentle transition was observed with a $\mathrm{pH}$ transition midpoint around 4.9 , which was successfully modeled when the uptake of protons was set to 1 . This value was equal to that used when modeling acid-base 
equilibria for monomers, and it reflects an acid-base equilibria lacking cooperative effects [50]. Therefore, we propose that this transition is caused by the protonation of cytosine bases located at the loops $\left(\mathrm{pK}_{\mathrm{a}}\right.$ around 4.5), and that are not involved in cytosine-cytosine ${ }^{+}$base pairs. Therefore, the overall $i$-motif structure was maintained. The presence of several $i$-motif structures over a $\mathrm{pH}$ range has been described previously for similar sequences [51, 52]. Finally, at around $\mathrm{pH} 3$, the $i$-motif evolved to a new species, most of the bases of which are possibly protonated.

The results obtained with the hybrid-modeling approach showed an intermediate behavior with respect to those of the other two approaches. Hence, the resolution was carried out for the three species detected by SVD and, therefore, a model with three species was proposed. The resolved concentration profiles of the intermediate species appeared detected by the hard-modeling method (see Fig. 2c).

The three approaches fitted the data in a similar fashion. The $\mathrm{pK}_{\mathrm{a}}$ values obtained for the hard- and hybrid-modeling methods showed a similar associated error (see Table 3). In this data set (D6), only the concentration and spectral profiles for the most basic species were concordant between the three methods. The other spectral and concentration profiles resolved showed significant differences between methods. This observation indicates that, more information is required to establish the most reliable outcome with certainty.

Data sets simulating a ligand-DNA interaction

The concentration profiles calculated and pure spectra are shown in Fig. 3 and the values obtained for the equilibrium constants are summarized in Table 4. From these results, the behavior of the three resolution approaches can be compared.

The hard-modeling analysis of data set D7 was carried out by proposing a model with three species (D, L, and DL) with an initial estimation of the equilibrium constant of 1,000 . The softmodeling resolution was performed using the non-negativity and closure constraints. Finally, we applied the hybrid-modeling method using the non-negativity and the hardmodeling constraintswith the same model previously described for the pure hard-modeling approach.

Hard- and hybrid-modeling methods provided similar results (Table 4 and Fig. 3); an observation that was also confirmed by the dissimilarity values (Electronic Supplementary Material Table S2). In contrast, the results obtained with the soft-modeling method differed significantly and the concentration profiles for the free ligand and for the interaction species were not correctly recovered. This finding is reflected in the high dissimilarity values calculated for the concentration profile of the interaction species and for the spectra of the ligand (resolved 
as a mixture of the spectra of the free ligand and the interaction species) and in the equilibrium constant recovered from the concentration profiles (Table 4).

The concentration and spectra profiles calculated for D8 are shown in Fig. 3c and d. Hard- and hybrid modeling methods showed a good resolution, while that obtained with the soft-modeling method was poor (especially for the ligand and the DL2 species). The values calculated for the equilibrium constants were similar except in the case of the soft-modeling approach.
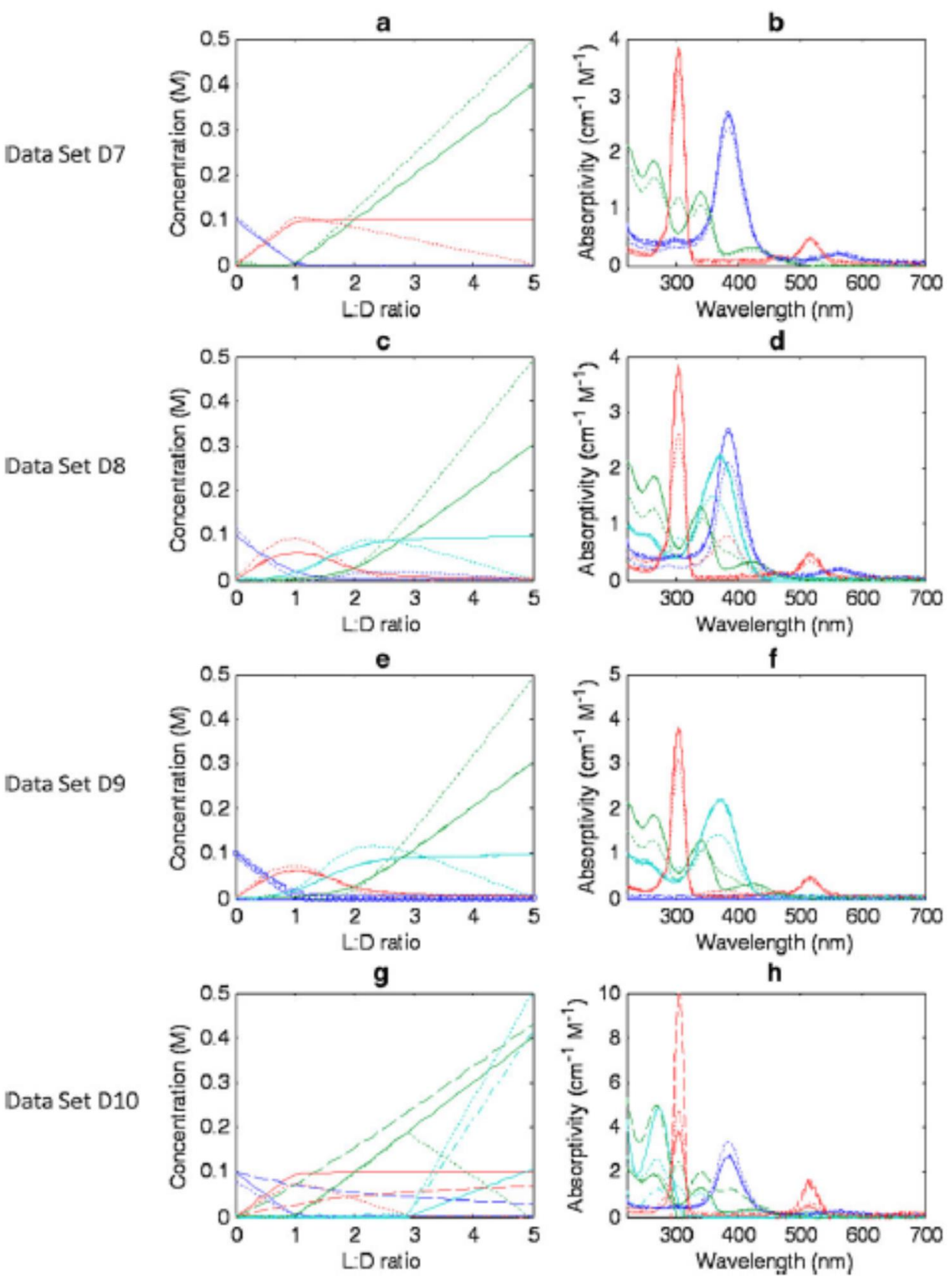

Fig. 3 Results from the analysis of the data sets simulating complexation equilibria. a Resolved concentration and b spectra profiles for data set D7. c Resolved concentration and d spectra profiles for data set D8. e Resolved concentration and f spectra profiles for data set D9. g Resolved concentration and $\mathrm{h}$ spectra profiles for data set D10. solid lines simulated profiles of spectroscopically active species, circle lines simulated profiles of spectroscopically inactive species, dashed lines hard-modeling profiles, dotted lines soft-modeling profiles, dash-dot lines hybrid-modeling profiles. 


\begin{tabular}{|c|c|c|c|c|}
\hline $\begin{array}{l}\text { Data } \\
\text { set }\end{array}$ & $\begin{array}{l}\text { Equilibrium } \\
\text { consant }\end{array}$ & $\begin{array}{l}\text { Hard- } \\
\text { modeling }\end{array}$ & $\begin{array}{l}\text { Soft- } \\
\text { modeling }\end{array}$ & $\begin{array}{l}\text { Hybrid- } \\
\text { modeling }\end{array}$ \\
\hline D7 & $K_{1}\left(\mathrm{M}^{-1}\right)$ & $2,062(55)$ & $2,410(890)$ & $2,057(61)$ \\
\hline \multirow[t]{2}{*}{ D8 } & $K_{1}\left(\mathrm{M}^{-1}\right)$ & $1,003(15)$ & $1,941(210)$ & $992(6)$ \\
\hline & $K_{2}\left(\mathrm{M}^{-1}\right)$ & $98(2)$ & $130(26)$ & $98(1)$ \\
\hline \multirow[t]{2}{*}{ D9 } & $K_{1}\left(\mathrm{M}^{-1}\right)$ & $985(22)$ & - & $981(46)$ \\
\hline & $K_{2}\left(\mathrm{M}^{-1}\right)$ & $102(3)$ & $398(149)$ & $91(2)$ \\
\hline D10 & $K_{1}\left(\mathrm{M}^{-1}\right)$ & $2,898(964)$ & $3,728(1760)$ & $2,020(57)$ \\
\hline
\end{tabular}

"Equilihrium ocnstant values obtained from soft modeling method MCRALS) ane cakulated from the concentration of exh species in the resolval concentration profiles at moleratios in which the species present in the considerod quilibriun has a significant oncentration value

The hard-modeling of the D9 data set was carried out using the same model as in D8 but indicating that the $\mathrm{D}$ species does not absorb. The soft-modeling analysis was performed for the three species detected in the SVD analysis and the resolution using the hybrid approach was also done for these three species but postulating a hard model with four species (D, L, DL, and DL2). The concentration and spectra profiles recovered are shown in Fig. 3e and f. These results were similar to those obtained in the analysis of the D8 data set. Only the pure soft-modeling method did not achieve a proper resolution of the data. This was due to the ambiguity in the resolution of the L and DL2 at higher concentration ratios under the constraints applied. In this case, the presence of an optically inactive species did not seem to hinder the goodness of the resolution by the hard- and hybrid-modeling methods.

Finally, the results obtained for the most difficult data set D10 are shown (Fig. 3g and h). A similar model to that used for D7 was applied in the hard-modeling (three species: D, L, and DL) because the interfering species could not be explicitly included in the model. The softmodeling approach was performed for four species (detected by SVD) and applying the same constraints as before. In the case of hybrid-modeling, only three of the four species considered were included in the model. Once again, the soft-modeling approach failed to properly resolve the data despite partial recovery of the interfering species. In contrast, the results obtained by the hard- and hybrid-modeling methods were clearly differentiated. The formed failed in the resolution of the data as can be seen in the value obtained for the equilibrium constant $(2,898$ $\left.\mathrm{M}^{\overline{1}}{ }^{1}\right)$ in relation to the theoretical value $\left(2,000 \mathrm{M}^{\overline{1}}\right)$. In addition, neither did the resolved spectra profiles coincide with those used in the simulation (see Fig. 3h and Electronic Supplementary Material Table S2). However, the hybrid approach showed satisfactory performance because the hard-modeling constraint allowed the proper resolution of the ligand-DNA interaction process (as in the analysis ofD7) and the interfering species was modeled by the ALS optimization under the non-negativity constraints. However, we observed a difference in the intensity of the 
profiles recovered for the interfering species and the profiles used for the simulation. This difference is attributed to the difficulties to define natural constraints that fulfill the interfering species (only non-negativity constraint for both concentration and spectral profiles was applied).

On the basis of the analysis of these simulated data sets, we wish to draw attention to several aspects. First, special mention is given to the difficulties observed for the soft-modeling method to achieve a good resolution of the data difficulties arise because the optimization algorithm tends to obtain pure species at the beginning and end of the experiment. However, this difficulty of the soft-modeling resolution can be easily overcome by applying more constraints (for instance, selectivity constraints that ensure that the concentration of the complex does not decrease at high ligand concentrations or the addition of information such as the pure spectrum of the ligand). Furthermore, we have shown that the hard- and hybrid-modeling methods behave similarly when the complexity of the system is not high. In this case, the results are totally comparable. However, when the complexity increases (for instance with the presence of an interfering species) the results obtained by the hybrid approach are significantly better when modeling the known chemical process with the hard model and the unknown variance with the soft model.

Experimental data sets corresponding to DNA-ligand interaction equilibria

Finally, two experimental data sets were analyzed in which the interaction of the krasG1 and krasC1 sequences with the model ligand TMPyP4 was studied. In both cases, the experiments were carried out at $\mathrm{pH}$ values in which the oligonucleotide showed an organized structure: $\mathrm{pH} 7$ for krasG1 sequence and $\mathrm{pH} 5$ for krasC1. In both cases, the interaction between the porphyrin and the oligonucleotide was denoted by a red shift of the Soret band of TMPyP4 (see Fig. 4a and b). First, the interaction of TMPyP4 with krasG1 was studied by means of the soft-modeling method. SVD detected three main species and the ALS optimization allowed the calculation of their concentration and spectra profiles (Fig. 4). These three species were assigned to the krasG1 oligonucleotide (whose spectrum showed a band centered around 250 $\mathrm{nm}$ and an increase in its concentration upon the experiment), the free TMPyP4 (whose spectrum showed a Soret band at $422 \mathrm{~nm}$ and a decrease in its concentration profile), and the interaction species (whose spectrum shows a characteristic peak at $440 \mathrm{~nm}$ and a concentration profile that shows a increase at the beginning of the experiment). Although the soft-modeling method allowed clear identification of the species, it is difficult to ensure the stoichiometry of the complex.

Fig. 4 Results from the analysis of the mole-ratio experiments. A Resolved concentration and b spectra profiles for the krasG1:TMPyP4 data set (D11). Inset detail of the resolved spectra of the free TMPyP4 
ligand (green line) and the DNA:TMPyP4 complex (red line) c) Resolved concentration and d spectra profiles for the krasC1:TMPyP4 data set (D12). Inset: Detail of the resolved spectra of the free TMPyP4 ligand (green line) and the DNA:TMPyP4 complex (red line). Legend: (dashed lines) hard-modeling profiles, (dotted lines) soft-modeling profiles, (dash-dot lines) hybrid-modeling profiles.
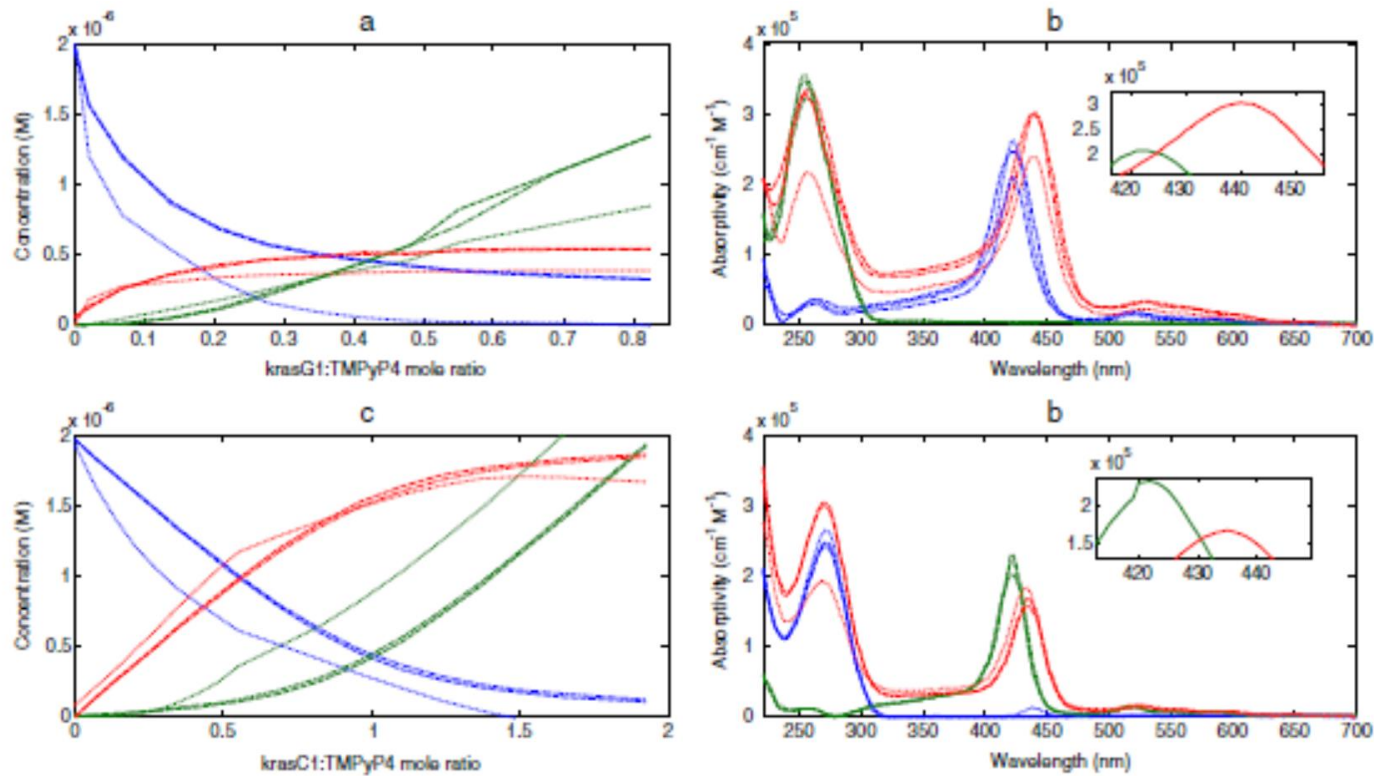

We performed hard-modeling defining a model involving three species: DNA, ligand, and one interaction species. Several stoichiometries for the interaction species were tested. Finally, the best results were obtained when a 1:3 (DNA:TMPyP4) stoichiometry was proposed (see Electronic Supplementary Material Figure S5b to observe the fitting of the calculated data to the experimental data at $445 \mathrm{~nm}$ ). The calculated value for the logarithm of the overall equilibrium constant was $19.1 \pm 0.3 \mathrm{M}^{i 3}$. The resolved spectral profiles (Fig. $4 \mathrm{~b}$ ) also showed the experimentally observed red shift in the maximum of absorbance of TMPyP4, characteristic of the interaction between an oligonucleotide and the porphyrin [53-55]. Finally, the hybrid approach, with a logarithm of the equilibrium constant of $18.9 \pm 0.4 \mathrm{M}^{\overline{1} 3}$, provided results similar (Fig. 4a and $\mathrm{b}$ ) to those obtained by the hard-modeling method.

Our results are consistent with those of previous studies found in the literature. Thus, Cogoi and Xodo [24, 25] examined this interaction between krasG1 and TMPyP4 and concluded that there are several types of possible interactions by end-stacking to the external Gtetrads and electrostatically to the bases of the larger loops. This conclusion was confirmed later by Arora and Maiti, who identified three possible interaction sites in similar G-quadruplex structures [53]. Therefore, the large magnitude of the red shift that we observed in the Soret region (from $422 \mathrm{~nm}$ of the free TMPyP4 to $441 \mathrm{~nm}$ of the complex, see inset in Fig. $4 \mathrm{~b}$ ) indicates a strong interaction between the G-quadruplex structure and the porphyrin. It can be concluded that, in this case, the interaction observed is not only electrostatic but there is also a stacking phenomena between TMPyP4 and krasG1. 
Finally, we report on the results of the interaction between krasC1 and TMPyP4. In this case, only three species were detected by means of SVD and, therefore, the soft-modeling resolution allowed the calculation of a set of concentration and spectral profiles, which were related to krasC1, TMPyP4, and the interaction species (Fig. 4c and d). Again the spectra profile recovered for the interaction species showed a clear red shift in the Soret region, thus reflecting the interaction between the oligonucleotide and the porphyrin (although the magnitude of this shift was smaller than for krasG1 case). In addition, the concentration profile of the complex krasC1: TMPyP4 showed a less steep slope than for the krasG1: TMPyP4 complex. This observation may reflect a smaller number of porphyrin molecules in the interaction species. This hypothesis was also confirmed in the absorbance profile at $445 \mathrm{~nm}$ (Electronic Supplementary Material Figure S4d), which had a less pronounced profile than that for the interaction between krasG1 and TMPyP4.

Thus, a hard-modeling analysis defining a model with three species and a stoichiometry for the interaction species equal to 1:1 (krasC1:TMPyP4) was performed. The calculated logarithm of equilibrium constant was $7.0 \pm 0.2 \mathrm{M}^{\top}$. The resolved spectra were similar to those obtained with the soft-modeling method. Similar results were obtained with the hybridmodeling method (logarithm of the equilibrium constant of $6.9 \pm 0.3 \mathrm{M}^{i}$ ).

These results are also concordant with previous studies reported in the literature [51, 52, $56,57]$. Those studies also observed the interaction between the $i$-motif structure and the TMPyP4 porphyrin. The inset of Fig. $4 d$ shows that the magnitude of the red shift caused by the formation of the complex between the krasC1 and TMPyP4 is significantly smaller than in krasG1 (12 nm for krasC1 and $19 \mathrm{~nm}$ for krasG1). This difference may be associated with the type of the interaction between the i-motif structure and the porphyrin, which in this case, could only be electrostatic. However, this type of structure is not as widely studied as the previous Gquadruplex structure. Consequently, few studies have addressed the possible stoichiometries of the complexes formed.

Conclusions

Here, we compared the performance of three chemometric tools to study the equilibria of biomolecules in solution, specifically, acid-base and ligand-DNA interaction equilibria. In both cases, the application of pure soft- and pure hard-modeling methods were found to be complementary. However, on the basis of our results, we propose that a hybrid approach, which allows exploitation of the advantages of both soft- and hard-modeling, is more efficient that the separate use of these approaches. Thus, like a soft-modeling method, the hybrid approach shows flexibility in the analysis (for instance, modeling an unknown interference or a complex baseline 
drift) while also allowing the proposal of a chemical model for the species involved in the equilibrium, as does a pure hard-modeling method.

Acknowledgments. This research was supported by the Spanish Ministerio de Ciencia e Innovación (grant number CTQ2009-11572) and the Generalitat de Catalunya (grant number 2009-SGR-45). Thanks go to Andrea Barba, Irene Giner, and Agnieszka StřpieFFUniversity of Barcelona) for carrying out part of the experimental work.

\section{References}

1. Brown SD, Tauler R, Walczak B (eds) (2009) Comprehensive chemometrics: chemical and biochemical data analysis, vol 2. Elsevier, Amsterdam

2. Massart DL, Vandegiste BGM, Buydens LMC, de Jong S, Lewi PJ, Smeyers-Verbeke J (1997) Handbook of chemometrics and qualimetrics. Elsevier, Oxford

3. Malinowski ER, Howery DG (2002) Factor analysis in chemistry, 3rd edn. Wiley, New York

4. Maeder M, Zuberbühler AD (1990) Nonlinear least-squares fitting of multivariate absorption data. Anal Chem 62(20):2220-2224

5. Dyson RM, Jandanklang P, Maeder M, Mason CJ, Whitson A (1999) New developments for the numerical analysis of spectrophotometric titrations. Polyhedron 18(25):3227-3232

6. Molloy KJ, Maeder M, Schumacher MM (1999) Hard modelling of spectroscopic measurements. Applications in non-ideal industrial reaction systems. Chemom Intell Lab Syst 46(2):221-230

7. Jiang JH, Ozaki Y (2002) Self-modeling curve resolution (SMCR): principles, techniques, and applications. Appl Spectrosc Rev 37(3):321-345

8. Lawton WH, Sylvestre EA (1971) Self modeling curve resolution. Technometrics 13(3):617633

9. De Juan A, Maeder M, Martínez M, Tauler R (2001) Application of a novel resolution approach combining soft- and hardmodelling features to investigate temperature-dependent kinetic processes. Anal Chim Acta 442(2):337-350

10. Tauler R, Smilde A, Kowalski B (1995) Selectivity, local rank, 3- way data-analysis and ambiguity in multivariate curve resolution. J Chemometr 9(1):31-58

11. Tauler R (1995) Multivariate curve resolution applied to second order data. Chemom Intell Lab Syst 30(1):133-146

12. De Juan A, Vander Heyden Y, Tauler R, Massart DL (1997) Assessment of new constraints applied to the alternating least squares method. Anal Chim Acta 346(3):307-318 
13. Jaumot J, Gargallo R, De Juan A, Tauler R (2005) A graphical user-friendly interface for MCR-ALS: a new tool for multivariate curve resolution in Matlab. Chemom Intell Lab Syst 76(1):101- 110

14. Jaumot J, Tauler R (2010) MCR-bands: a user friendly matlab program for the evaluation of rotation ambiguities in multivariate curve resolution. Chemom Intell Lab Syst 103(2):96-107

15. Tauler R (2001) Calculation of maximum and minimum band boundaries of feasible solutions for species profiles obtained by multivariate curve resolution. J Chemometr 15(8):627-646

16. Abdollahi H, Maeder M, Tauler R (2009) Calculation and meaning of feasible band boundaries in multivariate curve

resolution of a two-component system. Anal Chem 81(6):2115- 2122

17. De Juan A, Maeder M, Martínez M, Tauler R (2000) Combining hard- and soft-modelling to solve kinetic problems. Chemom Intell Lab Syst 54(2):123-141

18. Diewok J, De Juan A, Maeder M, Tauler R, Lendl B (2003) Application of a combination of hard and soft modeling for equilibrium systems to the quantitative analysis of $\mathrm{pH}$-modulated mixture samples. Anal Chem 75(3):641-647

19. Gemperline PJ, Cash E (2003) Advantages of soft versus hard constraints in self-modeling curve resolution problems. Alternating least squares with penalty functions. Anal Chem 75(16):4236-4243

20. Jaumot J, Gemperline PJ, Stang A (2005) Non-negativity constraints for elimination of multiple solutions in fitting of multivariate kinetic models to spectroscopic data. J Chemometr 19(2):97-106

21. Amigo JM, de Juan A, Coello J, Maspoch S (2006) A mixed hardand soft-modelling approach for the quantitative determination of oxipurines and uric acid in human urine. Anal Chim Acta 567 (2):236-244

22. Barbacid M (1987) Ras genes. Annu Rev Biochem 56:779-827

23. Malumbres M, Barbacid M (2003) Ras oncogenes: the first 30 years. Nat Rev Cancer $3(6): 459-465$

24. Cogoi S, Paramasivam M, Spolaore B, Xodo LE (2008) Structural polymorphism within a regulatory element of the human kras promoter: formation of g4-DNA recognized by nuclear proteins. Nucleic Acids Res 36(11):3765-3780

25. Cogoi S, Xodo LE (2006) G-quadruplex formation within the promoter of the kras protooncogene and its effect on transcription. Nucleic Acids Res 34(9):2536-2549

26. Neidle S, Balasubramanian S (2006) Quadruplex nucleic acids, 1st edn. RSC, Cambridge 
27. Simonsson T (2001) G-quadruplex DNA structures-variations on a theme. Biol Chem $382(4): 621-628$

28. Ahmed S, Kintanar A, Henderson E (1994) Human telomeric Cstrand tetraplexes. Nat Struct Biol 1(2):83-88

29. Gehring K, Leroy JL, Gueron M (1993) A tetrameric DNA structure with protonated cytosine cytosine base pairs. Nature 363 (6429):561-565

30. Gueron M, Leroy JL (2000) The i-motif in nucleic acids. Curr Opin Struct Biol 10(3):326331

31. Allawi HT, Santalucia J Jr (1997) Thermodynamics and NMR of internal GA·T mismatches in DNA. Biochemistry 36(34):10581- 10594

32. Dyson RM, Kaderli S, Lawrance GA, Maeder M, Zuberbühler AD (1997) Second order global analysis: the evaluation of series of spectrophotometric titrations for improved determination of equilibrium constants. Anal Chim Acta 353(2 3):381-393

33. De Juan A, Tauler R (2003) Chemometrics applied to unravel multicomponent processes and mixtures: revisiting latest trends in multivariate resolution. Anal Chim Acta 500(1-2):195210

34. Windig W, Guilment J (1991) Interactive self-modeling mixture analysis. Anal Chem 63(14):1425-1432

35. Muñoz G, de Juan A (2007) pH- and time-dependent hemoglobin transitions: a case study for process modelling. Anal Chim Acta 595(1-2 SPEC. ISS):198-208

36. Jaumot J, Menezes JC, Tauler R (2006) Quality assessment of the results obtained by multivariate curve resolution analysis of multiple runs of gasoline blending processes. $\mathrm{J}$ Chemometr 20 (1-2):54-67

37. Mathworks T (2008) Matlab release 2008a Natick, MA, US

38. Golub GH, Van Loan CF (1996) Matrix computations, 3rd edn. John Hopkins University Press, Baltimore

39. Bloomfield VA, Crothers DM, Tinoco I (2000) Nucleic acids: structures, properties, and functions

40. Mergny JL, Phan AT, Lacroix L (1998) Following G-quartet formation by UVspectroscopy. FEBS Lett 435(1):74-78

41. Bucek P, Jaumot J, Aviñó A, Eritja R, Gargallo R (2009) pHmodulated Watson-Crick duplex-quadruplex equilibria of guanine-rich and cytosine-rich DNA sequences 140 base pairs upstream of the c-kit transcription initiation site. Chem Eur J 15 (46):12663-12671

42. Leroy JL, Guéron M, Mergny JL, Hélène C (1994) Intramolecular folding of a fragment of the cytosine-rich strand of telomeric DNA into an i-motif. Nucleic Acids Res 22(9):1600-1606 
43. Mergny JL, Lacroix L, Han X, Leroy JL, Helene C (1995) Intramolecular folding of pyrimidine oligodeoxynucleotides into a i-DNA motif. J Am Chem Soc 117(35):8887-8898

44. Mathur V, Verma A, Maiti S, Chowdhury S (2004) Thermodynamics of i-tetraplex formation in the nuclease hypersensitive element of human c-myc promoter. Biochem Biophys Res Commun 320(4):1220-1227

45. Esmaili N, Leroy JL (2005) I-motif solution structure and dynamics of the d(AACCCC) and d(CCCCAA) tetrahymena telomeric repeats. Nucleic Acids Res 33(1):213-224

46. Zhou J, Wei C, Jia G, Wang X, Feng Z, Li C (2010) Formation of i-motif structure at neutral and slightly alkaline ph. Mol Biosyst 6 (3):580-586

47. Hill AV (1910) Proceedings of the physiological society: the possible effects of the aggregation of the molecules of huemoglobinon its dissociation curves. J Physiol 40(Suppl):ivii

48. Voet D, Voet JG (1995) Biochemistry, 3rd edn. Wiley, New York

49. Manzini G, Yathindra N, Xodo LE (1994) Evidence for intramolecularly folded i-DNA structures in biologically relevant ccc-repeat sequences. Nucleic Acids Res 22(22):4634-4640

50. Gargallo R, Tauler R, Izquierdo-Ridorsa A (1996) Influence of selectivity and polyelectrolyte effects on the performance of soft-modelling and hard-modelling approaches applied to the study of acid-base equilibria of polyelectrolytes by spectrometric titrations. Anal Chim Acta 331(3):195-205

51. Kumar P, Verma A, Maiti S, Gargallo R, Chowdhury S (2005) Tetraplex DNA transitions within the human c-myc promoter detected by multivariate curve resolution of fluorescence resonance energy transfer. Biochemistry 44(50):16426-16434

52. Khan N, Aviñó A, Tauler R, González C, Eritja R, Gargallo R (2007) Solution equilibria of the i-motif-forming region upstream of the b-cell lymphoma-2 p1 promoter. Biochimie 89(12):1562- 1572

53. Arora A, Maiti S (2008) Effect of loop orientation on quadruplex- TMPyP4 interaction. J Phys Chem B 112(27):8151-8159

54. Haq I, Trent JO, Chowdhry BZ, Jenkins TC (1999) Intercalative g-tetraplex stabilization of telomeric DNA by a cationic porphyrin. J Am Chem Soc 121(9):1768-1779

55. del Toro M, Bucek P, Aviñó A, Jaumot J, González C, Eritja R, Gargallo R (2009) Targeting the g-quadruplex-forming region near the p1 promoter in the human bcl-2 gene with the cationic porphyrin tmpyp4 and with the complementary c-rich strand. Biochimie 91(7):894902 
56. Fedoroff OY, Rangan A, Chemeris VV, Hurley LH (2000) Cationic porphyrins promote the formation of i-motif DNA and bind peripherally by a nonintercalative mechanism. Biochemistry 39(49):15083-15090

57. Simonsson T, Pribylova M, Vorlickova M (2000) A nuclease hypersensitive element in the human c-myc promoter adopts several distinct i-tetraplex structures. Biochem Biophys Res Commun 278(1):158-166. 


\section{Supplementary Material}

Table S1. Dissimilarity values obtained for the acid-base simulated data sets.

\begin{tabular}{|c|c|c|c|c|c|c|c|}
\hline & \multicolumn{6}{|c|}{ Dissimilarity values } \\
\hline & & \multicolumn{2}{|c|}{ Hard-modeling } & \multicolumn{2}{|c|}{ Soft-modeling } & \multicolumn{2}{|c|}{ Hybrid-modeling } \\
\hline & & Conc. & Spectra & Conc. & Spectra & Conc. & Spectra \\
\hline \multirow{4}{*}{ D1 } & Species 1 & 0.0009 & 0.0144 & 0.0351 & 0.0934 & 0.0023 & 0.0146 \\
\hline & Species 2 & 0.0010 & 0.0114 & 0.0932 & 0.0496 & 0.0022 & 0.0115 \\
\hline & Species 3 & 0.0010 & 0.0072 & 0.0440 & 0.0134 & 0.0023 & 0.0071 \\
\hline & Species 4 & 0.0005 & 0.0093 & 0.0308 & 0.0200 & 0.0013 & 0.0094 \\
\hline \multirow{5}{*}{ D2 } & Species 1 & 0.0021 & 0.0146 & 0.1009 & 0.0925 & 0.0034 & 0.0148 \\
\hline & Species 2 & 0.0020 & 0.0133 & 0.0881 & 0.0516 & 0.0038 & 0.0121 \\
\hline & Species 3 & 0.0146 & 0.0143 & 0.0856 & 0.0440 & 0.0007 & 0.0201 \\
\hline & Species 4 & 0.0138 & 0.2129 & 0.4522 & 0.3115 & 0.0005 & 0.1429 \\
\hline & Interference & - & - & 0.8065 & 0.9640 & 0.4625 & 0.0916 \\
\hline \multirow{4}{*}{ D3 } & Species 1 & 0.0002 & 0.0100 & 0.0434 & 0.0960 & 0.0051 & 0.0101 \\
\hline & Species 2 & 0.0006 & 0.0076 & 0.0941 & 0.0195 & 0.0086 & 0.0078 \\
\hline & Species 3 & - & - & - & - & - & - \\
\hline & Species 4 & 0.0002 & 0.0058 & 0.0137 & 0.0075 & 0.0086 & 0.0056 \\
\hline \multirow{5}{*}{ D4 } & Species 1 & 0.0040 & 0.0118 & 0.1645 & 0.0931 & 0.0014 & 0.0122 \\
\hline & Species 2 & 0.0037 & 0.0114 & 0.0989 & 0.0160 & 0.0129 & 0.0103 \\
\hline & Species 3 & - & - & - & - & - & - \\
\hline & Species 4 & 0.0244 & 0.2121 & 0.0244 & 0.2364 & 0.0122 & 0.1925 \\
\hline & Interference & - & - & 0.9025 & 0.2737 & 0.8153 & 0.0908 \\
\hline
\end{tabular}


Table S2. Dissimilarity values obtained for the complexation simulated data sets.

\begin{tabular}{|c|c|c|c|c|c|c|c|}
\hline & \multicolumn{6}{|c|}{ Dissimilarity } \\
\hline & & \multicolumn{2}{|c|}{ Hard-modeling } & \multicolumn{2}{|c|}{ Soft-modeling } & \multicolumn{2}{|c|}{ Hybrid-modeling } \\
\hline & & Conc. & Spectra & Conc. & Conc. & Spectra & Conc. \\
\hline \multirow{3}{*}{ D7 } & Species D & 0.0009 & 0.0403 & 0.0302 & 0.0659 & 0.0008 & 0.0209 \\
\hline & Species L & 0.0001 & 0.0052 & 0.0157 & 0.2474 & 0.0001 & 0.0043 \\
\hline & Species DL & 0.0009 & 0.0110 & 0.9630 & 0.0382 & 0.0008 & 0.0093 \\
\hline \multirow{4}{*}{ D8 } & Species D & 0.0014 & 0.0333 & 0.4076 & 0.0970 & 0.0003 & 0.0194 \\
\hline & Species L & 0.0005 & 0.0086 & 0.0370 & 0.2342 & 0.0005 & 0.0074 \\
\hline & Species DL & 0.0020 & 0.0257 & 0.1772 & 0.3962 & 0.0023 & 0.0203 \\
\hline & Species DL2 & 0.0013 & 0.0181 & 0.8258 & 0.3228 & 0.0011 & 0.0153 \\
\hline \multirow{4}{*}{ D9 } & Species D & - & - & - & - & - & - \\
\hline & Species L & 0.0005 & 0.0088 & 0.0651 & 0.2374 & 0.0036 & 0.0089 \\
\hline & Species DL & 0.0019 & 0.0277 & 0.2064 & 0.0553 & 0.0136 & 0.0214 \\
\hline & Species DL2 & 0.0014 & 0.0182 & 0.8306 & 0.2514 & 0.0078 & 0.0233 \\
\hline \multirow{4}{*}{ D10 } & Species D & 0.0110 & 0.0619 & 0.0344 & 0.0577 & 0.0004 & 0.0321 \\
\hline & Species L & 0.2738 & 0.0308 & 0.9488 & 0.4580 & 0.0001 & 0.0126 \\
\hline & Species DL & 0.2276 & 0.0296 & 0.9997 & 0.0415 & 0.0004 & 0.0215 \\
\hline & Interference & - & - & 0.0125 & 0.4192 & 0.0084 & 0.0184 \\
\hline
\end{tabular}


Figure S1. Building up of the simulated acid-base data sets. (a) Simulated concentration and (b) Spectra profiles for data set D1. (c) D1 simulated data after the addition of noise. (d) Simulated concentration and (e) Spectra profiles for data set D2. (f) D2 simulated data after the addition of noise. (g) Simulated concentration and (h) Spectra profiles for data set D3. (i) D3 simulated data after the addition of noise. (j) Simulated concentration and (k) Spectra profiles for data set D4. (l) D4 simulated data after the addition of noise. Legend: (solid lines) simulated profiles of spectroscopically active species, (dashed lines) simulated profiles of spectroscopically inactive species.
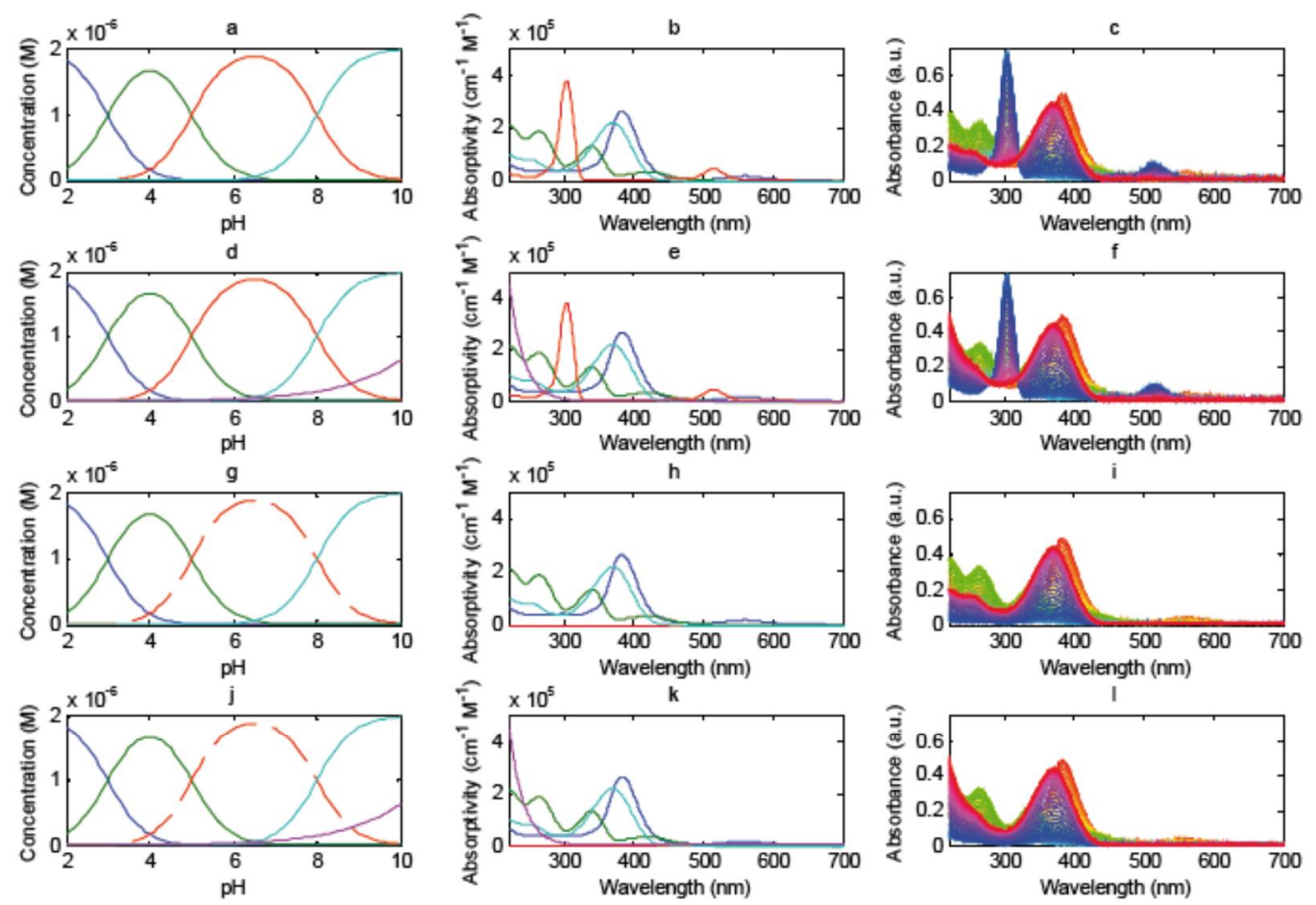
Figure S2. Building up of the simulated ligand-DNA complexation data. (a) Simulated concentration and (b) Spectra profiles for data set D7. (c) D7 simulated data after the addition of noise. (d) Simulated concentration and (e) Spectra profiles for data set D8. (f) D8 simulated data after the addition of noise. (g) Simulated concentration and (h) Spectra profiles for data set D9. (i) D9 simulated data after the addition of noise. (j) Simulated concentration and (k) Spectra profiles for data set D10. (1) D10 simulated data after the addition of noise. Legend: (solid lines) simulated profiles of spectroscopically active species, (dashed lines) simulated profiles of spectroscopically inactive species.
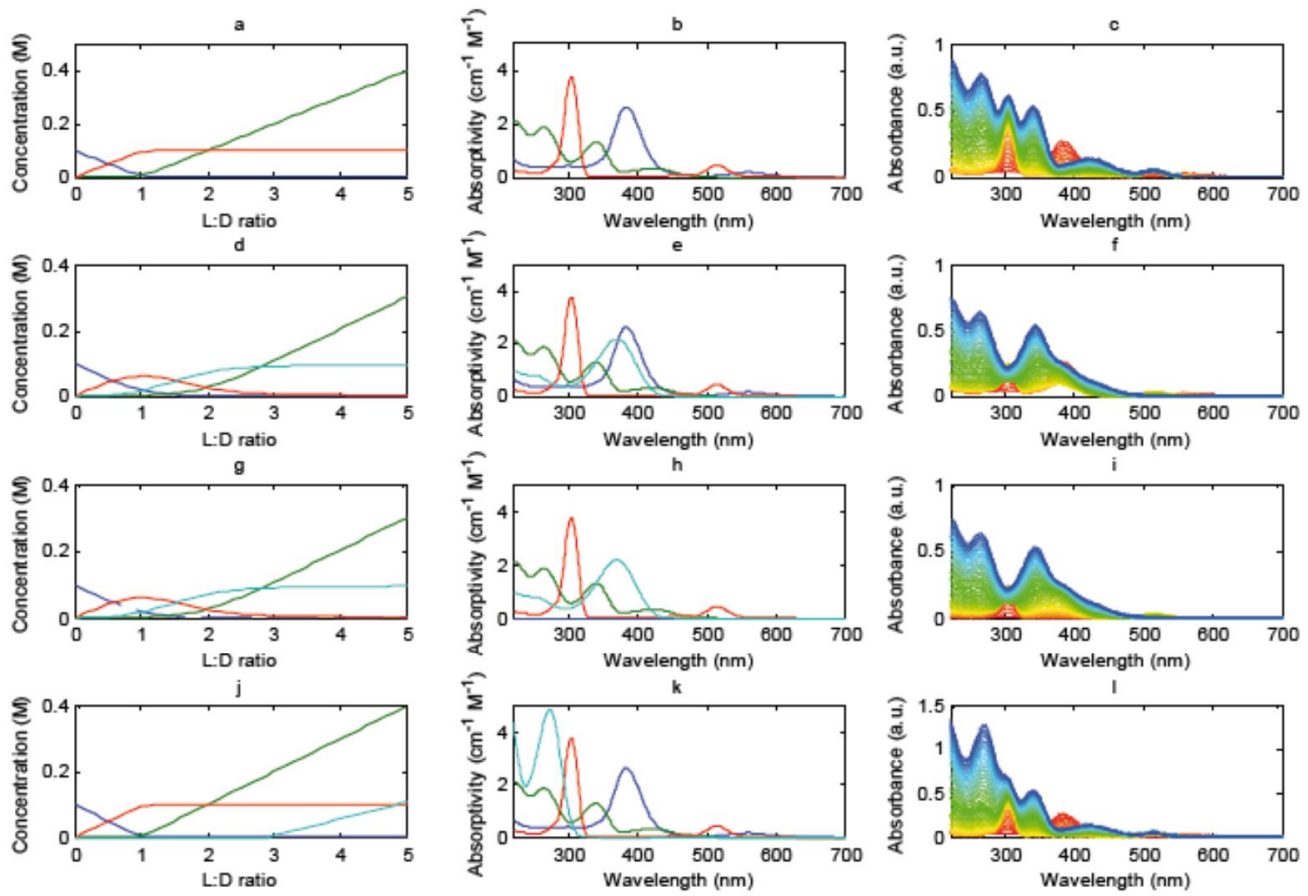
Figure S3. Band boundaries of the MCR-ALS resolved profiles in the analysis of data set D1. (a) Concentration profiles and (b) Spectra. Legend: (solid lines) resolved MCRALS profiles, (dashed lines) minimum and maximum band boundaries of the resolved profiles.
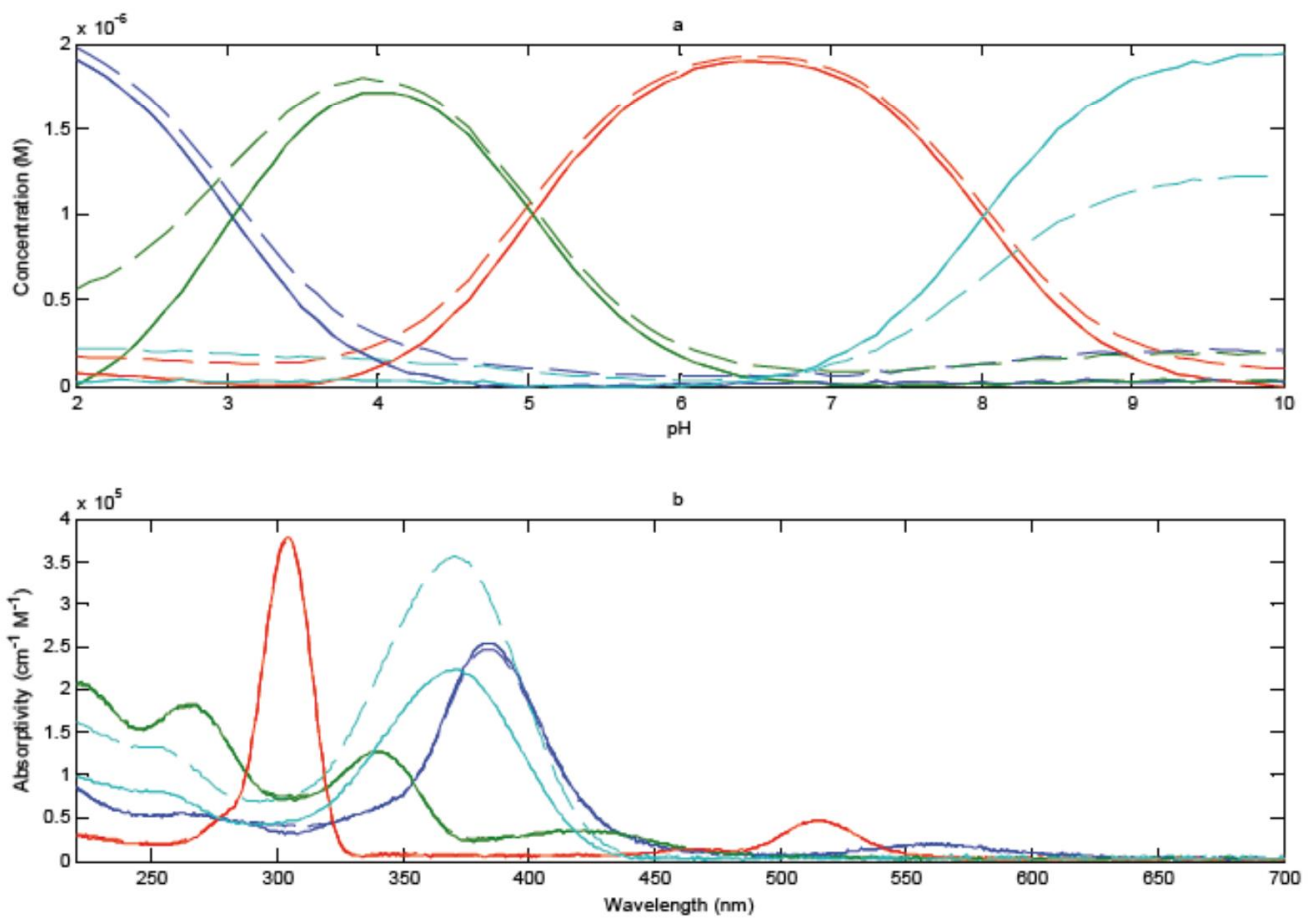
Figure S4. Fitting of the experimental acid-base data by means of the hard-modelling method. (a) Spectra obtained upon the $\mathrm{pH}$ titration of the krasG1 sequence. (b) Fitting of the experimental data by the proposed model at $295 \mathrm{~nm}$. (c) Spectra obtained upon the titration of the krasC1 sequence. (d) Fitting of the experimental data by the proposed model at $295 \mathrm{~nm}$. Legend: (x symbol) measured value of absorbance at the selected wavelength, (solid lines) fitted value of absorbance at the selected wavelength.
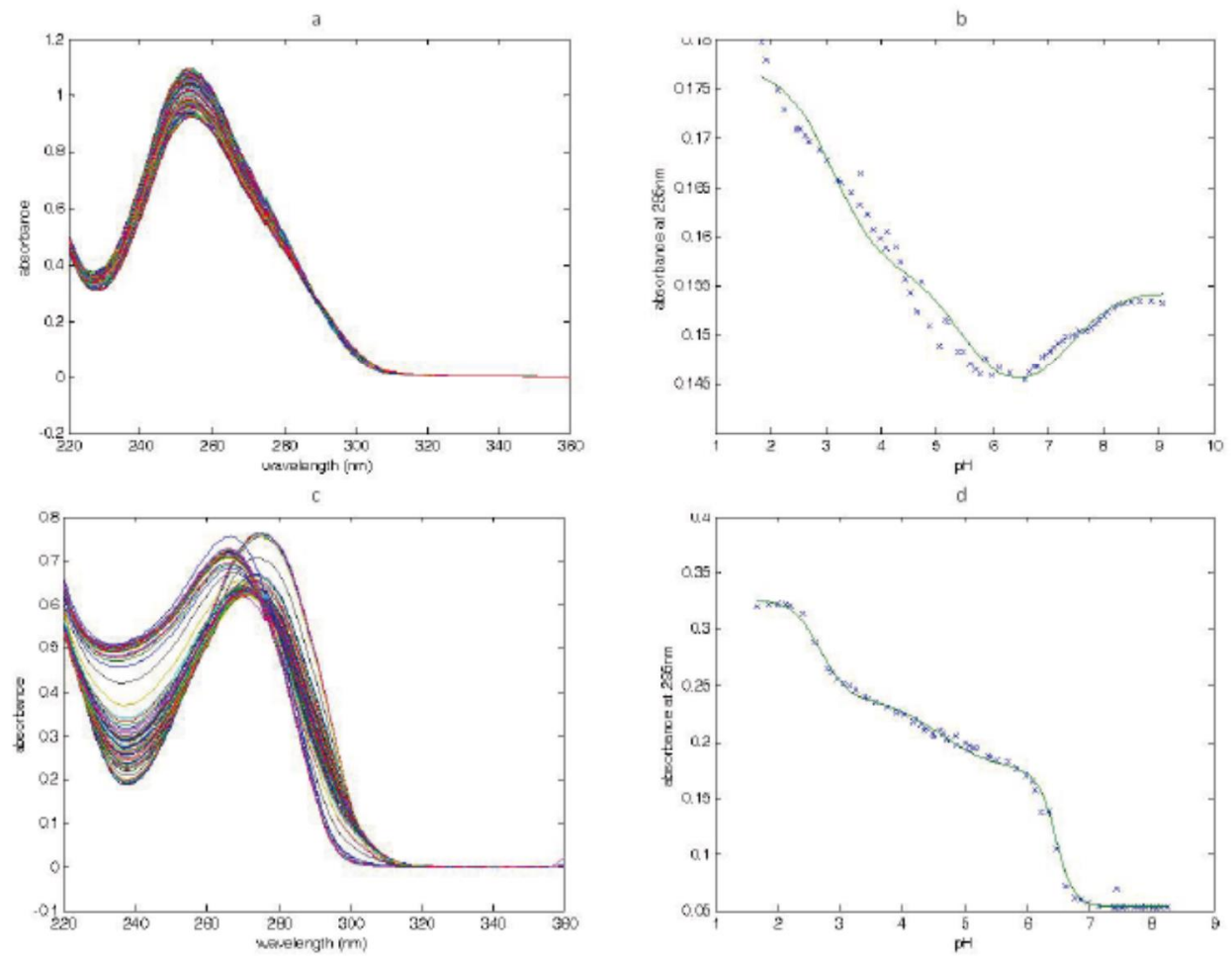
Figure S5. Fitting of the experimental complexation data by means of the hardmodelling method. (a) Spectra obtained upon the mole-ratio experiment between the krasG1 sequence and the TMPyP4 porphyrin. (b) Fitting of the experimental data by the proposed model at $445 \mathrm{~nm}$. (c) Spectra obtained upon the mole-ratio experiment between the krasC1 sequence and the TMPyP4 porphyrin. (d) Fitting of the experimental data by the proposed model at $445 \mathrm{~nm}$. Legend: ( $\mathrm{x}$ symbol) measured value of absorbance at the selected wavelength, (solid lines) fitted value of absorbance at the selected wavelength.
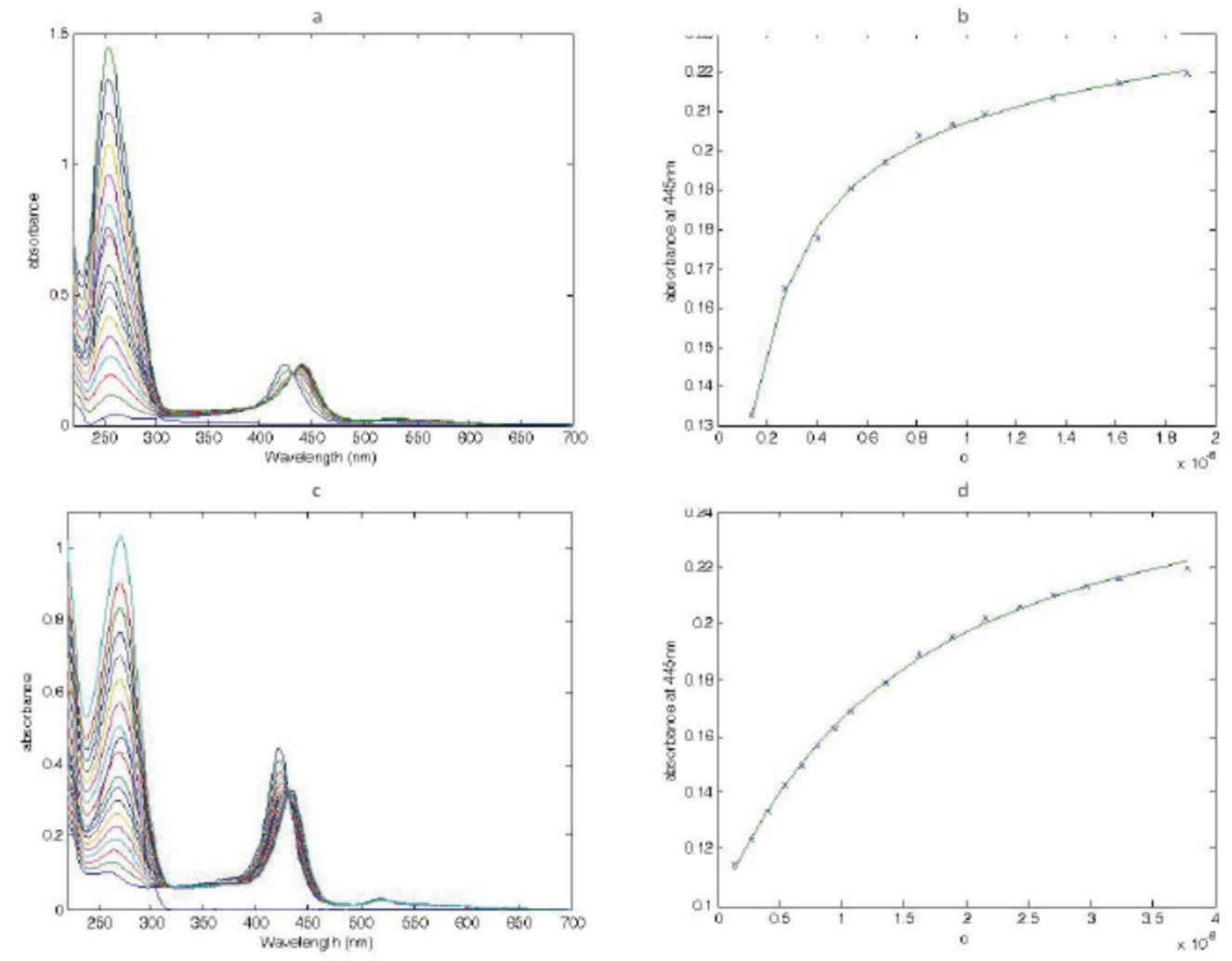